\title{
A New Approach to Solve the Low-lying States of the Schroedinger Equation ${ }^{1}$
}

\author{
T. D. Lee \\ Physics Department, Columbia University, New York, NY 10027 \\ China Center of Advanced Science and Technology , \\ (CCAST-World Laboratory), P.O. Box 8730, \\ Beijing 100080, People's Republic of China
}

\begin{abstract}
We review a new iterative procedure to solve the low-lying states of the Schroedinger equation, done in collaboration with Richard Friedberg. For the groundstate energy, the $n^{\text {th }}$ order iterative energy is bounded by a finite limit, independent of $n$; thereby it avoids some of the inherent difficulties faced by the usual perturbative series expansions. For a fairly large class of problems, this new procedure can be proved to give convergent iterative solutions. These convergent solutions include the long standing difficult problem of a quartic potential with either symmetric or asymmetric minima.
\end{abstract}

\footnotetext{
${ }^{1}$ This research was supported in part by the U.S. Department of Energy Grant DE-FG0292ER-40699
} 


\section{Importance of Low-lying States}

It is a common belief that most of natural phenomena should be described by solutions of the Schroedinger equation. Take the nonrelativistic Schroedinger equation of a system of charged particles with Coulomb interactions. In principle, this single $\mathrm{N}$-dimensional second order linear partial differential equation contains in its solutions all information about many disciplines: atomic and molecular physics (except for small relativistic corrections), condensed matter physics, chemistry and biology. Furthermore, for most of these applications we need only knowledge about its low-lying states. For these low-lying states, actually we do have very good information about their wave functions when the Coulomb interaction is strong; i.e., when the wave amplitude is large. How come that we still have great difficulties in solving these equations? Quite often, the challenge lies in how to handle the large number of configurations when the interaction is weak.

In quantum chromodynamics (QCD) and quantum electrodynamics (QED) the tasks are even more difficult since we do not have any exact solution. Besides the very promising lattice and other numerical calculations, we rely mostly on perturbative expansions. Yet, such expansion often leads to a divergent series with zero radius of convergence, as exemplified by the perturbative calculation of the gyromagnetic ratio $g$ of the muon[1-3]:

$$
\begin{aligned}
\frac{1}{2}(g-2)_{\mu}= & \frac{1}{2} \frac{\alpha}{\pi}+0.765857376\left(\frac{\alpha}{\pi}\right)^{2} \\
& +24.05050898\left(\frac{\alpha}{\pi}\right)^{3}+131.0\left(\frac{\alpha}{\pi}\right)^{4} \\
& +677(40)\left(\frac{\alpha}{\pi}\right)^{5}+\cdots
\end{aligned}
$$

where $\alpha$ is the fine-structure constant. The coefficients are all positive, and each successive one becomes larger and larger. There are good reasons [4] to believe that the vacuum state of QED would be unstable against pair creations if $\alpha$ could be analytically continued to the negative region. Thus, the difficulty of perturbative series seems to be again closely related to our inability to solve the low-lying states of the corresponding Schroedinger equation. In QCD, because of the existence of instanton configurations, similar serious problems also exist for its vacuum and other low-lying states. 
This situation may be illustrated by the following one dimensional problem with a quartic potential of degenerate minima. The Schroedinger equation can be written as

$$
(T+V-E) \psi=0
$$

where $T=-\frac{1}{2} \frac{d^{2}}{d x^{2}}$ and

$$
V(x)=\frac{1}{2} g^{2}\left(x^{2}-a^{2}\right)^{2} .
$$

An alternative form of the same problem may be obtained by setting $q \equiv$ $\sqrt{2 g a}(a-x)$, so that the Hamiltonian becomes

$$
H=T+V \equiv 2 g a \mathcal{H}
$$

with

$$
\mathcal{H}=-\frac{1}{2} \frac{d^{2}}{d q^{2}}+\frac{1}{2} q^{2}(1-e q)^{2},
$$

in which $\mathcal{H}, q$ and $e=1 / \sqrt{8 g a^{3}}$ are all dimensionless. The perturbative expression of the groundstate energy $E$ is[5-14]

$$
E=g a-\frac{1}{4 a^{2}}-\frac{9}{64} \frac{1}{g a^{5}}-\frac{89}{512} \frac{1}{g^{2} a^{8}}-O\left(\frac{1}{g^{3} a^{11}}\right),
$$

or, in terms of the dimensionless coupling $e^{2}$

$$
E=2 g a\left[\frac{1}{2}-e^{2}-\frac{9}{2} e^{4}-\frac{89}{2} e^{6}-\cdots\right],
$$

which has a similar characteristics as the $(g-2)$ expansion (1.1).

In this one-dimensional case, from (1.5), one sees that for $e^{2}$ negative, $\mathcal{H}$ has no groundstate; this explains why the power series (1.7) is divergent. For $e^{2}=1 / 8 g a^{3}$ positive, it is also possible to trace the origin of the difficulty. The corresponding perturbative series of the groundstate wave function $\psi(x)$ may be written as

$$
\psi(x)=e^{-g S_{0}-S_{1}-g^{-1} S_{2}-g^{-2} S_{3}-\cdots} .
$$


Since the potential $V(x)$ has two degenerate minima at $x= \pm a$, there are two different expansions depending on the normalization condition:

$$
\psi(a)=1, \quad \text { or } \quad \psi(-a)=1 .
$$

In either case, the perturbative series is divergent for any $g$. However, for $g$ large, the wave function $\psi(x)$ can be approximately described by the first two terms of these expansions. For $x>0$, using the normalization $\psi(a)=1$, we find

$$
e^{-g S_{0}-S_{1}}=\frac{2 a}{a+x} e^{-\frac{g}{3}(a-x)^{2}(2 a+x)},
$$

and for $x<0$ with $\psi(-a)=1$,

$$
e^{-g S_{0}-S_{1}}=\frac{2 a}{a-x} e^{-\frac{g}{3}(a+x)^{2}(2 a-x)} .
$$

Unlike $\psi(x)$, neither expression has a zero derivative at $x=0$. Furthermore, each carries a spurious pole-term, at $x=-a$ for (1.10) and $x=a$ for (1.11). In addition, if one stays with the perturbative series (1.8), each successive higher order expansion accentuates further these spurious pole terms. This suggests that we must not follow the high order perturbative expressions, especially in the region $x$ near 0 , when $\psi(x)$ is exponentially small.

In all these problems, it is not difficult to construct a trial function $\phi$ such that

$$
\phi \approx \psi \quad \text { when } \psi \text { is large, }
$$

and with the correct symmetry properties of $\psi$ (e.g., $\phi(x)=\phi(-x)$ for the quartic potential problem (1.2)-(1.3)). The question is how to device a convergent procedure that can lead from the trial function $\phi$ to the correct $\psi$. In the following we will discuss such a method. 


\section{The New Method}

As examples of the types of Schroedinger equations that we are interested, consider the bosonic component of QED or QCD in the axial gauge, or a system of nonrelativistic particles. In either of these cases the kinetic energy $T$ is a quadratic function of momentum operators. Through a linear transformation of the relevant coordinate variables, the Hamiltonian $H$ can be written as

$$
H=T+V(q)
$$

where

$$
q=\left(q_{1}, q_{2}, \cdots, q_{N}\right)
$$

and

$$
T=-\frac{1}{2} \sum_{i=1}^{N} \partial^{2} / \partial q_{i}^{2}=-\frac{1}{2} \nabla^{2}
$$

with $N=\infty$ in the case of a field theory. Let $\psi(q)$ be the groundstate determined by

$$
(H-E) \psi=0 \text {. }
$$

To derive $\psi(q)$, we proceed as follows [15-19]:

1. Construct a good trial function $\phi(q)$, so that

$$
\phi(q) \approx \psi(q)
$$

in regions when $\psi(q)$ is expected to be large. An example of how to construct such a trial function is given in Appendix A.

2. Construct $U(q)-E_{0}$ by differentiating $\phi(q)$ :

$$
U(q)-E_{0} \equiv\left(\frac{1}{2} \nabla^{2} \phi\right) / \phi .
$$

Define

$$
H_{0}=-\frac{1}{2} \nabla^{2}+U(q)
$$


We have

$$
\left(H_{0}-E_{0}\right) \phi(q)=0
$$

where the constant $E_{0}$ may be defined by requiring, say, the potential energy part $U(q)$ of $H_{0}$ to satisfy at $q=\infty$

$$
U(\infty)=0 .
$$

Alternatively, in problems like the quartic potential (1.3), the constant $E_{0}$ can be more conveniently determined by requiring

$$
\text { minimum of } U(q)=0 .
$$

(Since our method deals only with the operator $H_{0}-E_{0}$, it is independent of the particular way to define $E_{0}$.) Introduce the differences $h$ and $\mathcal{E}$ by

$$
H_{0}-H=U(q)-V(q) \equiv h(q)
$$

and

$$
E_{0}-E \equiv \mathcal{E} \text {. }
$$

The original Schroedinger equation (2.4) can be written as

$$
\left(H_{0}-E_{0}\right) \psi(q)=(h-\mathcal{E}) \psi(q) .
$$

For the discussions of the groundstate, we take both

$$
\psi(q) \text { and } \phi(q) \text { to be real and positive. }
$$

Multiplying (2.13) by $\phi(q)$ and (2.8) by $\psi(q)$, then taking their difference, we have

$$
-\frac{1}{2} \nabla \cdot(\phi \nabla \psi-\psi \nabla \phi)=(h-\mathcal{E}) \phi \psi .
$$

The integration of (2.15) over all space gives $\int(h-\mathcal{E}) \phi \psi d^{N} q=0$; therefore,

$$
\mathcal{E}=\int h \phi \psi d^{N} q / \int \phi \psi d^{N} q
$$


It is convenient to introduce

$$
f(q)=\psi(q) / \phi(q)
$$

(2.16) becomes then

$$
\mathcal{E}=\int h(q) \phi^{2}(q) f(q) d^{N} q / \int \phi^{2}(q) f(q) d^{N} q .
$$

3. We propose to solve the Schroedinger equation (2.13) by an iterative process, defined by

$$
\left(H_{0}-E_{0}\right) \psi_{n}(q)=\left(h(q)-\mathcal{E}_{n}\right) \psi_{n-1}(q)
$$

with $n=1,2, \cdots$ and for $n=0$,

$$
\psi_{0}(q)=\phi(q) .
$$

Multiplying (2.19) by $\phi(q)$ and $(\underline{2.8})$ by $\psi_{n}(q)$, then taking their difference, we derive

$$
-\frac{1}{2} \nabla \cdot\left(\phi \nabla \psi_{n}-\psi_{n} \nabla \phi\right)=\left(h-\mathcal{E}_{n}\right) \phi \psi_{n-1} .
$$

Analogous to (2.16), we have

$$
\mathcal{E}_{n}=\int h \phi \psi_{n-1} d^{N} q / \int \phi \psi_{n-1} d^{N} q .
$$

Note that in each step of the iteration from $n-1$ to $n$, for any particular solution $\psi_{n}^{0}(q)$ that satisfies (2.19), so is

$$
\psi_{n}(q)=\psi_{n}^{0}(q)+c_{n} \phi(q)
$$

also a solution where $c_{n}$ is an arbitrary constant. Since $\phi(q)$ is positive in accordance with (2.14), we can choose $c_{n}$ so that

$$
\psi_{n}(q) \quad \text { is positive everywhere. }
$$

Define

$$
f_{n}(q)=\psi_{n}(q) / \phi(q)
$$


and therefore (2.22) becomes

$$
\mathcal{E}_{n}=\int h \phi^{2} f_{n-1} d^{N} q / \int \phi^{2} f_{n-1} d^{N} q
$$

From (2.24), we have

$$
f_{n}(q) \text { positive. }
$$

As we shall see, a particularly convenient choice of the constant $c_{n}$ is to set the minimum of $f_{n}(q)$ to be 1 and therefore

$$
f_{n}(q) \geq 1 \text {. }
$$

From (2.26) and (2.27), it follows that $\mathcal{E}_{n}$ is bounded if $h(q)$ is bounded. Therefore, as $n \rightarrow \infty, \lim \mathcal{E}_{n}$ cannot be $\infty$. This avoids the type of divergence difficulties that appears in the example (1.7). It is therefore reasonable to expect that $\lim _{n \rightarrow \infty} \mathcal{E}_{n}$ should converge to the correct $\mathcal{E}$, at least when $h$ is small. As we shall see, for a fairly large class of problems, including the quartic potential (1.3), the convergence of $\mathcal{E}_{n}$ turns out to be independent of the magnitude of $h(q)$, provided that it is finite and satisfies some general conditions.

In this paper, we concentrate on the groundstate. Extensions to some lowlying states can be found in Ref.[17]. 


\section{An Electrostatic Analog}

In terms of the ratio $f_{n}=\psi_{n} / \phi$ introduced in (2.25), the $\mathrm{n}^{\text {th }}$ order iterative equation (2.21) can be written as

$$
-\frac{1}{2} \nabla \cdot\left(\phi^{2} \nabla f_{n}\right)=\sigma_{n}(q)
$$

where

$$
\sigma_{n}(q) \equiv\left(h(q)-\mathcal{E}_{n}\right) \phi^{2}(q) f_{n-1}(q)
$$

and when $n=0$,

$$
f_{0}(q)=1
$$

in accordance with (2.20). Assuming that $\psi_{n-1}(q)$ has already been solved, we can determine $\mathcal{E}_{n}$ through (2.22). Therefore, $\sigma_{n}(q)$ is a known function.

Consider a dielectric medium with a q-dependent dielectric constant, given by

$$
\kappa(q) \equiv \phi^{2}(q) .
$$

Interpret $\sigma_{n}(q)$ as an external electrostatic charge distribution, $\frac{1}{2} f_{n}$ the electrostatic potential, $-\frac{1}{2} \nabla f_{n}$ the electrostatic field and

$$
D_{n} \equiv-\frac{1}{2} \kappa \nabla f_{n}
$$

the corresponding displacement vector field. Thus (3.1) becomes

$$
\nabla \cdot D_{n}=\sigma_{n}
$$

the Maxwell equation for this electrostatic analog problem.

At infinity, $\phi(\infty)=0$. In accordance with (3.4) - (3.5), we also have $D_{n}(\infty)=0$. Hence the integration of (3.6) leads to the total external electrostatic charge to be also zero; i.e.,

$$
\int \sigma_{n}(q) d^{N} q=0
$$


which is the same result given by (2.26) for the determination of $\mathcal{E}_{n}$. Because the dielectric constant $\kappa(q)$ in this analog problem is zero at $q=\infty$, the dielectric media becomes a perfect dia-electric at $\infty$. Thus, the equation of zero total charge, given by (3.7), may serve as a much simplified model of charge confinement, analogous to color confinement in quantum chromodynamics.

We note that (3.1) can also be derived from a minimal principle by defining

$$
I\left(f_{n}(q)\right) \equiv \int\left\{\frac{1}{4} \kappa\left(\nabla f_{n}\right)^{2}-\sigma_{n} f_{n}\right\} d^{N} q .
$$

Because of (3.7), the functional $I\left(f_{n}(q)\right)$ is invariant under

$$
f_{n}(q) \rightarrow f_{n}(q)+\text { constant. }
$$

Since the quadratic part of $I\left(f_{n}(q)\right)$ is the integral of the positive definite $\frac{1}{4} \kappa\left(\nabla f_{n}\right)^{2}$, the curvature of $I\left(f_{n}(q)\right)$ in the functional space $f_{n}(q)$ is always positive. Hence, $I\left(f_{n}(q)\right)$ has a minimum, and that minimum determines a unique electrostatic field $-\frac{1}{2} \nabla f_{n}$, as we shall see. To establish the uniqueness, let us assume two different $\nabla f_{n}$, both satisfy (3.1), with the same $\kappa=\phi^{2}$ and the same $\sigma_{n}$; their difference would then satisfy (3.1) with a zero external charge distribution. For $\sigma_{n}=0$, the minimum of $I\left(f_{n}(q)\right)$ is clearly zero with the corresponding $\nabla f_{n}=0$. To derive $f_{n}(q)$ from $\nabla f_{n}$, there remains an additive constant at each iteration, as already noted in (2.23). As we shall discuss in the next section, this arbitrariness allows us the freedom to derive different types of convergent series.

We note that in this electrostatic analog, the problem depends only on two input-functions: $\phi^{2}(q)$ and the product

$$
h(q) \phi^{2}(q) \text {. }
$$

The original potential $V(q)$ no longer appears explicitly. Consider, e.g., a system of particles with electric charges. For configurations when the Coulomb energy $V(q)$ is attractive and near its singularity, we have good knowledge about the wave amplitude $\psi(q)$, which is large but regular. The same should be true for a good trial function $\phi(q)$. In accordance with (1.12), we expect

$$
h(q) \text { small when } \psi \text { is large. }
$$


For configurations when $\psi$ is small, so should be $\phi$. Thus, a good trial function $\phi$ would result in a small $h \phi^{2}$ everywhere even though $h$ may not.

When $N=1$, we may denote the coordinate (2.2) by a single $x$. Correspondingly (3.5) and (3.6) become

$$
D_{n}=-\frac{1}{2} \kappa \frac{d f_{n}}{d x}
$$

and

$$
\frac{d D_{n}}{d x}=\sigma_{n}(x)
$$

Since $D_{n}(\infty)=0$, we have

$$
D_{n}(x)=-\int_{x}^{\infty} \sigma_{n}(z) d z
$$

and therefore

$$
f_{n}(x)=f_{n}(\infty)-2 \int_{x}^{\infty} \phi^{-2}(y) d y \int_{y}^{\infty} \sigma_{n}(z) d z
$$

with

$$
\sigma_{n}(x)=\left(h(x)-\mathcal{E}_{n}\right) \phi^{2}(x) f_{n-1}(x)
$$

which satisfies

$$
\int_{-\infty}^{\infty} \sigma_{n}(x) d x=0
$$

Therefore,

$$
D_{n}(-\infty)=D_{n}(\infty)=0
$$

and

$$
f_{n}(\infty)-f_{n}(-\infty)=2 \int_{-\infty}^{\infty} \phi^{-2}(y) d y \int_{y}^{\infty} \sigma_{n}(z) d z .
$$

When $N>1$, for radially symmetric problems we have

$$
h=h(r), \quad \phi^{2}=\kappa(r)
$$


where

$$
r^{2}=q_{1}^{2}+q_{2}^{2}+\cdots+q_{N}^{2} .
$$

Likewise $\psi, \psi_{n}$ and $\sigma_{n}$ are all functions of $r$ only. The radial component of $D_{n}$ is

$$
\left(D_{n}\right)_{r}=-\frac{1}{2} \kappa(r) \frac{d f_{n}(r)}{d r} .
$$

Correspondingly, (3.6) becomes

$$
\frac{1}{r^{N-1}} \frac{d}{d r}\left(r^{N-1}\left(D_{n}\right)_{r}\right)=\sigma_{n}(r),
$$

with

$$
\sigma_{n}(r)=\left(h(r)-\mathcal{E}_{n}\right) \phi^{2}(r) f_{n-1}(r) .
$$

Hence,

$$
\left(D_{n}\right)_{r}=-\frac{1}{r^{N-1}} \int_{r}^{\infty} z^{N-1} \sigma_{n}(z) d z
$$

and on account of (3.22),

$$
f_{n}(r)=f_{n}(\infty)-\int_{r}^{\infty} \frac{2 d y}{y^{N-1} \phi^{2}(y)} \int_{y}^{\infty} z^{N-1} \sigma_{n}(z) d z .
$$

Assuming as $r \rightarrow \infty$,

$$
\phi^{2}(r) \rightarrow a e^{-b r^{l}}
$$

with $a, b$ and $l$ all positive constants. We assume

$$
l>2 .
$$

In accordance with (3.16), as $r \rightarrow \infty$

$$
\sigma_{n}(r) \rightarrow c e^{-b r^{l}}
$$

where

$$
c=\left(h(\infty)-\mathcal{E}_{n}\right) a f_{n-1}(\infty) .
$$


By partial integrations, we see that as $r \rightarrow \infty$,

$$
f_{n}(r) \rightarrow f_{n}(\infty)-\frac{2}{b} \frac{\left(h(\infty)-\mathcal{E}_{n}\right) f_{n-1}(\infty)}{l(l-2) r^{l-2}}\left[1+O\left(\frac{1}{b r^{l}}\right)\right] .
$$

When the dimension $N=1$, the same expression applies, with $r$ replaced by $x$.

Because of (3.7). in the $N>1$ radially symmetric case we have

$$
\int_{0}^{\infty} r^{N-1} \sigma_{n}(r) d r=0
$$

therefore (3.25) is identical to

$$
f_{n}(r)=f_{n}(0)-\int_{0}^{r} \frac{2 d y}{y^{N-1} \phi^{2}(y)} \int_{0}^{y} z^{N-1} \sigma_{n}(z) d z .
$$

When $N=1$ and $V(x)$ is an even function of $x$, (3.32) applies with the replacement of $r$ by $x$; if $V(x)$ is not an even function then we have, from (3.15) and (3.19),

$$
f_{n}(x)=f_{n}(-\infty)-2 \int_{-\infty}^{x} \phi^{-2}(y) d y \int_{-\infty}^{y} \sigma_{n}(z) d z .
$$

For an arbitrary $N>1$ dimensional radially non-symmetric problem, the general solution of the electrostatic analog problem can no longer be reduced to simple quadratures. Thus, numerical analysis may become important. In that case, the minimal principle using the functional $I(f(q))$ given by (3.8) may be of some practical use. 


\section{Hierarchy Theorem}

In this section we restrict our discussions to either (i) the $N$-dimensional radially symmetric case in which the functions $V$ in (2.1) and $h$ in (2.11) are

$$
V=V(r) \quad \text { and } \quad h=h(r)
$$

where $r^{2}=q_{1}^{2}+q_{2}^{2}+\cdots+q_{N}^{2}$ as in (3.21), or (ii) the one-dimensional case (3.12)-(3.17) with even functions

$$
V(x)=V(-x) \quad \text { and } \quad h(x)=h(-x) .
$$

In the latter case, we need only to consider the region

$$
x \equiv r \geq 0 .
$$

Thus, we need only to consider (i), since the $N$-dimensional radially symmetric solution (3.25) reduces to the one-dimensional case (3.15), with $N=1$ and $r$ replaced by $x$. Furthermore, we assume $h(r)$ to satisfy at all finite $r>0$,

$$
h(r)>0 \text { and } h^{\prime}(r)<0
$$

at infinity,

$$
h(\infty)=0 .
$$

Throughout the paper, ' denotes $\frac{d}{d r}$. An example of such $h(r)$ is given in Appendix A for the quartic potential problem (1.3).

In accordance with (3.25), each $n^{\text {th }}$ iterative solution $f_{n}(r)$ carries an independent additive constant. In the following, we differentiate two sets of sequences, labelled $A$ and $B$, satisfying different boundary conditions:

$$
f_{n}(\infty)=1 \text { for all } n, \text { in Case }(A)
$$

or

$$
f_{n}(0)=1 \quad \text { for all } n, \quad \text { in Case }(B) .
$$

Thus, by using (3.25) or (3.32) we have in Case $(A)$

$$
f_{n}(r)=1-2 \int_{r}^{\infty} y^{-N+1} \phi^{-2}(y) d y \int_{y}^{\infty} z^{N-1} \phi^{2}(z)\left(h(z)-\mathcal{E}_{n}\right) f_{n-1}(z) d z,
$$


and in Case $(B)$

$f_{n}(r)=1-2 \int_{0}^{r} y^{-N+1} \phi^{-2}(y) d y \int_{0}^{y} z^{N-1} \phi^{2}(z)\left(h(z)-\mathcal{E}_{n}\right) f_{n-1}(z) d z$.

In both cases, $\mathcal{E}_{n}$ is determined by the corresponding $f_{n-1}(r)$ through (2.22); i.e.,

$$
\mathcal{E}_{n}=\left[h f_{n-1}\right] /\left[f_{n-1}\right]
$$

in which $[F]$ of any function $F(r)$ is defined to be

$$
[F]=\int_{0}^{\infty} r^{N-1} \phi^{2}(r) F(r) d r .
$$

It is convenient to introduce $\chi(r)$ defined by

$$
\chi(r) \equiv r^{(N-1) / 2} \phi(r) .
$$

Thus, (4.10) becomes

$$
[F]=\int_{0}^{\infty} \chi^{2}(r) F(r) d r .
$$

Let $\rho_{n}(r), \mathcal{K}(r)$ and $\mathcal{D}_{n}(r)$ be related to $\sigma_{n}(r), \kappa(r)$ and $\left(D_{n}\right)_{r}$ of (3.2), (3.20) and (3.22) by

$$
\begin{gathered}
\rho_{n}(r)=r^{N-1} \sigma_{n}(r)=\left(h(r)-\mathcal{E}_{n}\right) \chi^{2}(r) f_{n-1}(r) \\
\mathcal{K}(r)=r^{N-1} \kappa(r)=\chi^{2}(r)
\end{gathered}
$$

and

$$
\mathcal{D}_{n}(r)=r^{N-1}\left(D_{n}\right)_{r}=-\frac{1}{2} \mathcal{K}(r) f_{n}^{\prime}(r) .
$$

Correspondingly, in Case $(A),(4.8 \mathrm{~A})$ can be written as

$$
f_{n}(r)=1-2 \int_{r}^{\infty} \chi^{-2}(y) d y \int_{y}^{\infty} \chi^{2}(z)\left(h(z)-\mathcal{E}_{n}\right) f_{n-1}(z) d z,
$$

and in Case $(B),(4.8 \mathrm{~B})$ becomes

$$
f_{n}(r)=1-2 \int_{0}^{r} \chi^{-2}(y) d y \int_{0}^{y} \chi^{2}(z)\left(h(z)-\mathcal{E}_{n}\right) f_{n-1}(z) d z .
$$


Because of (3.23), we have

$$
\mathcal{D}_{n}^{\prime}(r)=\rho_{n}(r)
$$

and therefore

$$
\mathcal{D}_{n}(r)=-\int_{r}^{\infty} \rho_{n}(z) d z=-\int_{r}^{\infty}\left(h(z)-\mathcal{E}_{n}\right) \chi^{2}(z) f_{n-1}(z) d z .
$$

From (3.7),

$$
\int_{0}^{\infty} \rho_{n}(r) d r=0
$$

which leads to

$$
\mathcal{D}_{n}(r)=\int_{0}^{r} \rho_{n}(z) d z=\int_{0}^{r}\left(h(z)-\mathcal{E}_{n}\right) \chi^{2}(z) f_{n-1}(z) d z .
$$

These two expressions of $\mathcal{D}_{n}(r)$, (4.18) and (4.20), are valid for both cases $(A)$ and $(B)$. Let $r_{n}$ be defined by

$$
h(r)-\mathcal{E}_{n}=0 \quad \text { at } \quad r=r_{n} .
$$

Since $h^{\prime}(r)<0$, (4.21) has one and only one solution, with $h(r)-\mathcal{E}_{n}$ negative for $r>r_{n}$ and positive for $r<r_{n}$. Thus, if

$$
f_{n-1}(r)>0
$$

for all $r>0$, we have from (4.18) and (4.20)

$$
\mathcal{D}_{n}(r)>0
$$

and therefore, on account of (4.15),

$$
f_{n}^{\prime}(r)<0 .
$$

In Case $(A)$, because of $f_{n}(\infty)=1$, (4.24) leads to

$$
f_{n}(0)>f_{n}(r)>f_{n}(\infty)=1 .
$$

Since for $n=0, f_{0}(r)=1$, (4.22) - (4.25A) are valid for $n=1$; by induction these expressions also hold for all $n$; in Case $(A)$, their validity imposes no 
restriction on the magnitude of $h(r)$. In Case $(B)$ we assume $h(r)$ to be not too large, so that $(4.16 \mathrm{~B})$ is consistent with

$$
f_{n}(r)>0 \quad \text { for all } r
$$

and therefore

$$
f_{n}(0)=1>f_{n}(r)>f_{n}(\infty)>0 .
$$

As we shall see, these two boundary conditions $(A)$ and $(B)$ produce sequences that have very different behavior. Yet, they also share a number of common properties.

Hierarchy Theorem $(A)$ With the boundary condition $f_{n}(\infty)=1$, we have for all $n$

$$
\mathcal{E}_{n+1}>\mathcal{E}_{n}
$$

and

$$
\frac{d}{d r}\left(\frac{f_{n+1}(r)}{f_{n}(r)}\right)<0 \quad \text { at any } \quad r>0 .
$$

Thus, the sequences $\left\{\mathcal{E}_{n}\right\}$ and $\left\{f_{n}(r)\right\}$ are all monotonic, with

$$
\mathcal{E}_{1}<\mathcal{E}_{2}<\mathcal{E}_{3}<\cdots
$$

and

$$
1<f_{1}(r)<f_{2}(r)<f_{3}(r)<\cdots
$$

at all finite $r$.

$(B)$ With the boundary condition $f_{n}(0)=1$, we have for all odd $n=2 m+1$ an ascending sequence

$$
\mathcal{E}_{1}<\mathcal{E}_{3}<\mathcal{E}_{5}<\cdots
$$

but for all even $n=2 m$, a descending sequence

$$
\mathcal{E}_{2}>\mathcal{E}_{4}>\mathcal{E}_{6}>\cdots
$$


furthermore, between any even $n=2 m$ and any odd $n=2 l+1$

$$
\mathcal{E}_{2 m}>\mathcal{E}_{2 l+1} \text {. }
$$

Likewise, at any $r$, for any even $n=2 m$

$$
\frac{d}{d r}\left(\frac{f_{2 m+1}(r)}{f_{2 m}(r)}\right)<0,
$$

whereas for any odd $n=2 l+1$

$$
\frac{d}{d r}\left(\frac{f_{2 l+2}(r)}{f_{2 l+1}(r)}\right)>0 .
$$

The proof of the hierarchy theorem is given in Appendix B. The hierarchy theorem was discussed in our earlier papers for one-dimensional problems $[18,19]$. The extension to the $N$-dimensional radially symmetric case is new. As we shall also discuss in Appendix $B$, the lowest eigenvalue $E$ of the Hamiltonian $T+V$ is the limit of the sequence $\left\{E_{n}\right\}$ with

$$
E_{n}=E_{0}-\mathcal{E}_{n}
$$

Thus, the boundary condition $f_{n}(\infty)=1$ yields a sequence, in accordance with (4.28),

$$
E_{1}>E_{2}>E_{3}>\cdots>E
$$

with each member $E_{n}$ an upper bound of $E$, similar to the usual variational method.

On the other hand, with the boundary condition $f_{n}(0)=1$, while the sequence of its odd members $n=2 l+1$ yields a similar one, like (4.36), with

$$
E_{1}>E_{3}>E_{5}>\cdots>E
$$

its even members $n=2 m$ satisfy

$$
E_{2}<E_{4}<E_{6}<\cdots<E .
$$

It is unusual to have an iterative sequence of lower bounds of the eigenvalue $E$. Together, these sequences may be quite efficient to pinpoint the limiting E.

In Appendix C, we discuss the comparison between the iterative solution and perturbative series for some simple examples. 


\section{Tribute}

This paper is based on my joint work with Richard Friedberg. It was a pleasure for me to present the main body of the paper at the Number and Nature Symposium held on December 17-18, 2004 at the Rockefeller University, as a celebration for Mitchell J. Feigenbaum's 60th birthday. In the Chinese tradition, sixty is very special. It signifies the successful completion of one's first cycle, and the fresh beginning of a new second cycle.

Because China has no indigenous religion, there is no origin of reference in the Chinese calender, nothing equivalent to $B C$ or $A D$. All Chinese years are labelled cyclically, modulus sixty.

In Chinese calender, each year is designated by two indices. The first index runs cyclically from one to ten, with each number represented by a different word shown in the first column of Chinese characters in Figure 1. We may translate the first character at the top of that column as alpha (for one) and the second character as beta (for two), then gamma (for three), . . until kappa (for ten); after kappa, we have alpha, beta, $\cdots$ again. The second column of Chinese characters has twelve words, each for a different animal. In Figure 1 , the year 1944, when Mitchell was born is designated (Alpha-Monkey), the next year 1945 is (Beta-Chicken), . .; ten years later, the first index for 1954 becomes again alpha, but the second index is horse; two years later for 1956, the first (number) index is gamma, but the second (animal) index becomes monkey again. It takes $\frac{1}{2} \cdot 10 \cdot 12=60$ years to complete the cycle. This year 2004 is like 1944, the Alpha-Monkey year and Mitchell is now in his new cycle, starting a new beginning.

the most beloved story in China is the book "Monkey" by Wu Cheng-En written in the 16th century. It is about an immortal Monkey-King of Chaos. The translation was by Arthur Waley (Figure 2) in 1943, perhaps anticipating that 1944 was the beginning of Mitchell's Alpha-Monkey cycle. To celebrate 2004, the beginning of Mitchell's newest cycle, Figure 3 is a drawing of mine, entitled "Monkey King the Thinker". All of us are looking forward to this new cycle with our very best wishes. 


\section{Figure Captions}

1. Chinese calender is cyclic, modulus sixty.

2. One of the most beloved novels in China

3. "Monkey King the Thinker" by T. D. Lee (2004) (color online) 


\section{Chinese Calender}

\begin{tabular}{|c|c|c|c|c|}
\hline (alpha) & $\phi$ & $\phi$ & (monkey) & 1944 \\
\hline (beta) & 己 & (i) & (chiken) & 45 \\
\hline (gamma) & 两 & $\frac{1}{x}$ & (dog) & 46 \\
\hline . & , & & & \\
\hline - & $\cdot$ & & & \\
\hline (kappa) & 烁 & 2 & (snake) & 53 \\
\hline (alpha) & $\phi$ & 7 & (horse) & 54 \\
\hline (beta ) & 2 & 末 & (sheep) & 55 \\
\hline \multirow[t]{4}{*}{ (gamma) } & 两 & $\phi$ & (monkey) & 56 \\
\hline & . & - & & \\
\hline & - & $\cdot$ & & \\
\hline & . & - & & \\
\hline (alpha) & 9 & $\phi$ & (monkey) & 2004 \\
\hline
\end{tabular}

Figure 1 


\section{Monkey}

\section{King of Chaos}

Written by Wu Cheng-En

(16 ${ }^{\text {th }}$ Century)

\section{Translated by Arthur Waley}

(1943)

Figure 2 


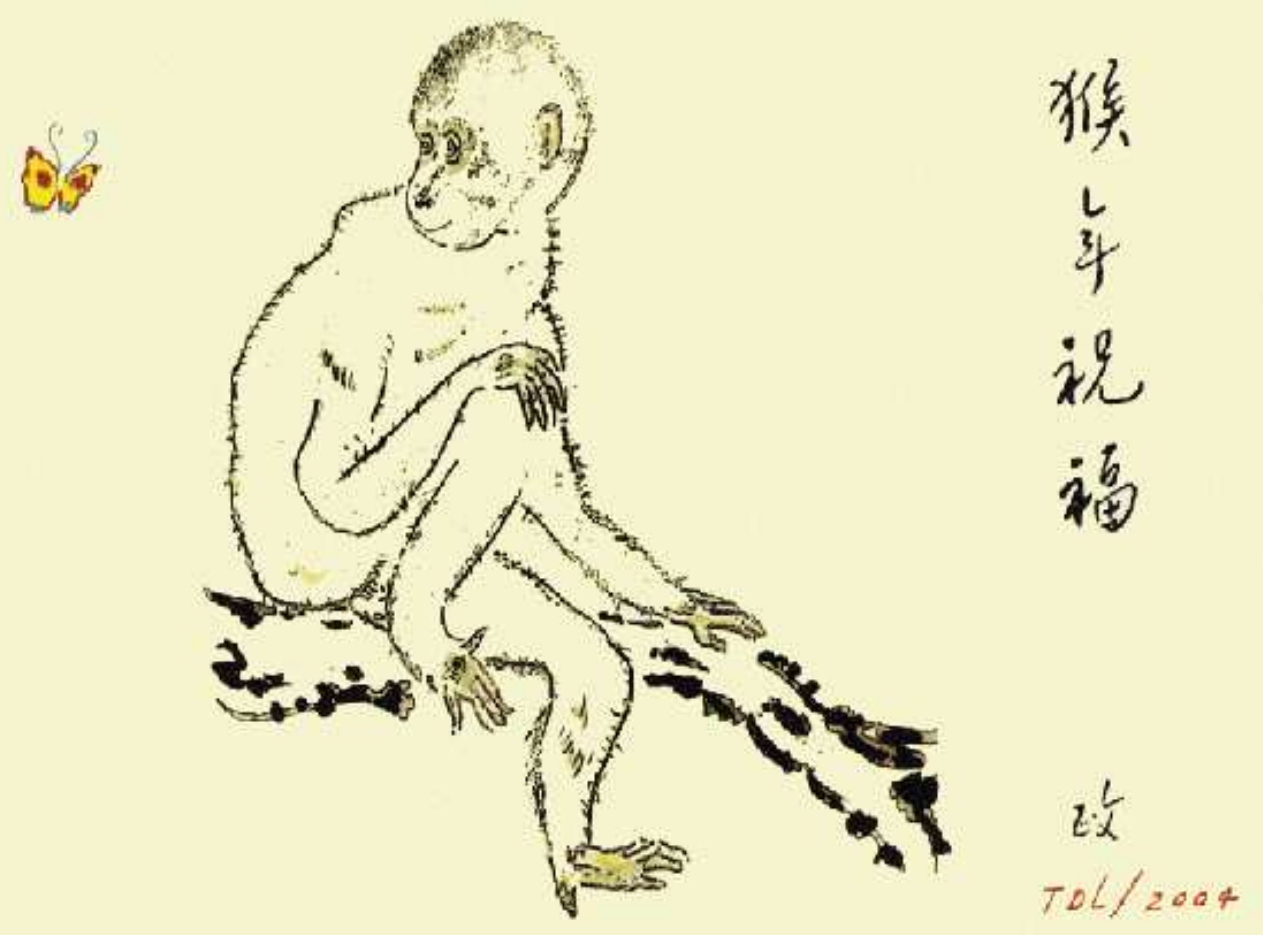

Monkey King The Thinker

Figure 3 


\section{References}

[1] W. Marciano, hep-ph/0411179

[2] M. Davier and W. Marciano, Annu. Rev. Nucl. Part. Sci. 54(2004), 115

[3] M. Passera, hep-ph/0411168

[4] F. Dyson (unpublished)

[5] A. M. Polyakov, Nucl.Phys. B121 (1977), 429

[6] G. 't Hooft, in: A. Zichichi, Erice(Eds.), The why's of subnuclear physics Plenum, New York, 1977

[7] E. Brezin, G. Parisi and J. Zinn-Justin, Phys.Rev. D16 (1977), 408

[8] J. Zinn-Justin, J.Math.Phys. 22 (1981), 511

[9] J. Zinn-Justin, Nucl.Phys. B192 (1981), 125

[10] J. Zinn-Justin, in: J.-D. Zuber, R. Stora (Eds.), Recent advances in field theory and statistical mechanics, Les Houches, session XXXIX, 1982

[11] J. Zinn-Justin, Private Communication

[12] Sidney Coleman, Aspects of Symmetry, Press Syndicate of the University of Cambridge, 1987

[13] E. Shuryak, Nucl.Phys. B302 (1988), 621

[14] S. V. Faleev and P. G. Silvestrov, Phys. Lett. A197 (1995), 372

[15] R. Friedberg, T. D. Lee and W. Q. Zhao

IL Nuovo Cimento A112 (1999), 1195

[16] R. Friedberg, T. D. Lee and W. Q. Zhao, Ann.Phys. 288 (2001), 52

[17] R. Friedberg, T. D. Lee, W. Q. Zhao and A. Cimenser Ann.Phys. 294 (2001), 67

[18] R. Friedberg and T. D. Lee, Ann.Phys. 308 (2003), 263

[19] R. Friedberg and T. D. Lee, Preprint quant-ph/0407207 Ann. Phys. (in press)

[20] See, e.g., Philip M. Morse and Herman Feshbach, Methods of Theoretical Physics, McGraw-Hill Brook Co. (1953) 


\section{Appendix A Construction of Trial Functions}

\section{A.1 A New Formulation of Perturbative Expansion}

As mentioned in Section 1, in many problems of interest, perturbative expansion lead to asymptotic series. Nevertheless, the first few terms of such an expansion could provide important insight to what a good trial function might be. For our purpose, a particularly convenient way is to follow the method developed in Refs.[15] and [16]. As we shall see, in this new method to each order of the perturbation, the wave function is always expressible in terms of a single line-integral in the $\mathrm{N}$-dimensional coordinate space, which can be readily used for the construction of the trial wave function.

We begin with the Hamiltonian $H$ in its standard form (2.1) $-(2.3)$. Assume $V(q)$ to be positive definite, and choose its minimum to be at $q=0$, with

$$
V(q) \geq V(0)=0 .
$$

Introduce a scale factor $g^{2}$ by writing

$$
V(q)=g^{2} v(q)
$$

and correspondingly

$$
\psi(q)=e^{-g S(q)} .
$$

Thus, the Schroedinger equation (2.4) becomes

$$
\left(-\frac{1}{2} \nabla^{2}+g^{2} v(q)\right) e^{-g S(q)}=E e^{-g S(q)}
$$

where, as before, $q$ denotes $q_{1}, q_{2}, \cdots, q_{N}$ and $\nabla$ the corresponding gradient operator. Hence $S(q)$ satisfies

$$
-\frac{1}{2} g^{2}(\nabla S)^{2}+\frac{1}{2} g \nabla^{2} S+g^{2} v=E .
$$

Considering the case of large $g$, we expand

$$
S(q)=S_{0}(q)+g^{-1} S_{1}(q)+g^{-2} S_{2}(q)+\cdots
$$

and

$$
E=g E_{0}+E_{1}+g^{-1} E_{2}+\cdots .
$$


Substituting (A.6) - (A.7) into (A.5) and equating the coefficients of $g^{-n}$ on both sides, we find

$$
\begin{aligned}
\left(\nabla S_{0}\right)^{2} & =2 v \\
\nabla S_{0} \cdot \nabla S_{1} & =\frac{1}{2} \nabla^{2} S_{0}-E_{0}, \\
\nabla S_{0} \cdot \nabla S_{2} & =\frac{1}{2}\left[\nabla^{2} S_{1}-\left(\nabla S_{1}\right)^{2}\right]-E_{1}, \\
\nabla S_{0} \cdot \nabla S_{3} & =\frac{1}{2}\left[\nabla^{2} S_{2}-2 \nabla S_{1} \cdot \nabla S_{2}\right]-E_{2},
\end{aligned}
$$

etc. In this way, the second order partial differential equation (A.5) is reduced to a series of first order partial differential equations (A.8). The first of this set of equations can be written as

$$
\frac{1}{2}\left[\nabla S_{0}(q)\right]^{2}-v(q)=0+.
$$

As noted in Ref.[15], this is precisely the Hamilton-Jacobi equation of a single particle with unit mass moving in a potential " $-v(q)$ " in the N-dimensional $q$-space. Since $q=0$ is the maximum of the classical potential energy function $-v(q)$, for any point $q \neq 0$ there is always a classical trajectory with a total energy $0+$, which begins from $q=0$ and ends at the other point $q \neq 0$, with $S_{0}(q)$ given by the corresponding classical action integral. Furthermore, $S_{0}(q)$ increases along the direction of the trajectory, which can be extended beyond the selected point $q \neq 0$, towards $\infty$. At infinity, it is easy to see that $S_{0}(q)=\infty$, and therefore the corresponding wave amplitude $e^{-g S_{0}(q)}$ is zero. To solve the second equation in (A.8), we note that, in accordance with (A.1) - (A.2) at $q=0, \nabla S_{0} \propto v^{\frac{1}{2}}(0)=0$. By requiring $S_{1}(q)$ to be analytic at $q=0$, we determine

$$
E_{0}=\frac{1}{2}\left(\nabla^{2} S_{0}\right)_{\text {at } q=0}
$$

It is convenient to consider the surface

$$
S_{0}(q)=\text { constant; }
$$

its normal is along the corresponding classical trajectory passing through $q$. Characterize each classical trajectory by the $S_{0}$-value along the trajectory and 
a set of $N-1$ angular variables

$$
\alpha=\left(\alpha_{1}(q), \alpha_{2}(q), \cdots, \alpha_{N-1}(q)\right),
$$

so that each $\alpha$ determines one classical trajectory with

$$
\nabla \alpha_{j} \cdot \nabla S_{0}=0
$$

where

$$
j=1,2, \cdots, N-1 .
$$

(As an example, we note that as $q \rightarrow 0, v(q) \rightarrow \frac{1}{2} \sum_{i} \omega_{i}^{2} q_{i}^{2}$ and therefore $S_{0} \rightarrow \frac{1}{2} \sum_{i} \omega_{i} q_{i}^{2}$. Consider the ellipsoidal surface $S_{0}=$ constant. For $S_{0}$ sufficiently small, each classical trajectory is normal to this ellipsoidal surface. A convenient choice of $\alpha$ could be simply any $N-1$ orthogonal parametric coordinates on the surface.) Each $\alpha$ designates one classical trajectory, and vice versa. Every $\left(S_{0}, \alpha\right)$ is mapped into a unique set $\left(q_{1}, q_{2}, \cdots, q_{N}\right)$ with $S_{0} \geq 0$ by construction. In what follows, we regard the points in the $q$-space as specified by the coordinates $\left(S_{0}, \alpha\right)$. Depending on the problem, the mapping $\left(q_{1}, q_{2}, \cdots, q_{N}\right) \rightarrow\left(S_{0}, \alpha\right)$ may or may not be one-to-one. We note that, for $q$ near 0 , different trajectories emanating from $q=0$ have to go along different directions, and therefore must associate with different $\alpha$. Later on, as $S_{0}$ increases each different trajectory retains its initially different $\alpha$-designation; consequently, using $\left(S_{0}, \alpha\right)$ as the primary coordinates, different trajectories never cross each other. The trouble-some complications of trajectory-crossing in $q$-space is automatically resolved by using $\left(S_{0}, \alpha\right)$ as coordinates. Keeping $\alpha$ fixed, the set of first order partial differential equations can be further reduced to a set of first order ordinary differential equations, which are readily solvable, as we shall see.

Write

$$
S_{1}(q)=S_{1}\left(S_{0}, \alpha\right)
$$

the second line of (A.8) becomes

$$
\left(\nabla S_{0}\right)^{2}\left(\frac{\partial S_{1}}{\partial S_{0}}\right)_{\alpha}=\frac{1}{2} \nabla^{2} S_{0}-E_{0}
$$


and leads to, besides (A.10), also

$$
S_{1}(q)=S_{1}\left(S_{0}, \alpha\right)=\int_{0}^{S_{0}} \frac{d S_{0}}{\left(\nabla S_{0}\right)^{2}}\left[\frac{1}{2} \nabla^{2} S_{0}-E_{0}\right],
$$

where the integration is taken along the classical trajectory of constant $\alpha$. Likewise, the third, fourth and other lines of (A.8) lead to

$$
\begin{gathered}
E_{1}=\frac{1}{2}\left[\nabla^{2} S_{1}-\left(\nabla S_{1}\right)^{2}\right] \text { at } q=0, \\
S_{2}(q)=S_{2}\left(S_{0}, \alpha\right)=\int_{0}^{S_{0}} \frac{d S_{0}}{\left(\nabla S_{0}\right)^{2}}\left\{\frac{1}{2}\left[\nabla^{2} S_{1}-\left(\nabla S_{1}\right)^{2}\right]-E_{1}\right\}, \\
E_{2}=\frac{1}{2}\left[\nabla^{2} S_{2}-2\left(\nabla S_{1}\right) \cdot\left(\nabla S_{2}\right)\right] \text { at } q=0, \\
S_{3}(q)=S_{3}\left(S_{0}, \alpha\right)=\int_{0}^{S_{0}} \frac{d S_{0}}{\left(\nabla S_{0}\right)^{2}}\left\{\frac{1}{2}\left[\nabla^{2} S_{2}-2\left(\nabla S_{1}\right) \cdot\left(\nabla S_{2}\right)\right]-E_{2}\right\},
\end{gathered}
$$

etc. These solutions give the convenient normalization convention at $q=0$,

$$
S(0)=0
$$

and

$$
e^{-S(0)}=1
$$

$\underline{\text { Remarks }}$

(i) As an example, consider an $\mathrm{N}$-dimensional harmonic oscillator with

$$
V(q)=\frac{g^{2}}{2}\left(q_{1}^{2}+q_{2}^{2}+\cdots+q_{N}^{2}\right) .
$$

From (A.2), one sees that the Hamilton-Jacobi equation (A.9) is for a particle moving in a potential given by

$$
-v(q)=-\frac{1}{2}\left(q_{1}^{2}+q_{2}^{2}+\cdots+q_{N}^{2}\right) .
$$


Thus, for any point $q \neq 0$ the classical trajectory of interest is simply a straight line connecting the origin and the specific point, with the action

$$
S_{0}(q)=\frac{1}{2}\left(q_{1}^{2}+q_{2}^{2}+\cdots+q_{N}^{2}\right) .
$$

The corresponding energy is, in accordance with (A.10),

$$
E_{0}=\frac{N}{2} .
$$

By using (A.8), one can readily show that $E_{1}=E_{2}=\cdots=0$ and $S_{1}=S_{2}=$ $\cdots=0$. The result is the well known exact answer with the groundstate wave function for the Schroedinger equation (A.4) given by

$$
e^{-g S(q)}=\exp \left[-\frac{g}{2}\left(q_{1}^{2}+q_{2}^{2}+\cdots+q_{N}^{2}\right)\right]
$$

and the corresponding energy

$$
E=\frac{N}{2} g .
$$

(ii) From this example, it is clear that the above expressions (A.6) - (A.8) are not the well-known WKB method. The new formalism uses $-v(q)$ as the potential for the Hamilton-Jacobi equation, and its "classical" trajectory carries a $0+$ energy; consequently, unlike the WKB method, there is no turning point along the classical trajectory, and the formalism is applicable to arbitrary dimensions.

\section{A.2 Trial Function for the Quantum Double-well Potential}

To illustrate how to construct a trial function, consider the quartic potential (1.3) in one dimension with degenerate minima. Set $a=1$, we have

$$
V(x)=\frac{1}{2} g^{2}\left(x^{2}-1\right)^{2} .
$$

The corresponding Schroedinger equation is

$$
\left(-\frac{1}{2} \frac{d^{2}}{d x^{2}}+\frac{1}{2} g^{2}\left(x^{2}-1\right)^{2}\right) \psi(x)=E \psi(x)
$$


where, as before, $\psi(x)=e^{-g S(x)}$ is the groundstate wave function and $E$ its energy. Using the expansions (A.6) - (A.7) and following the steps (A.8), (A.10) and (A.15) - (A.21), we find the well-known perturbative series

$S_{0}(x)=\frac{1}{3}(x-1)^{2}(x+2), \quad S_{1}(x)=\ln \frac{x+1}{2}, \quad S_{2}(x)=\frac{3}{16}-\frac{x+2}{4(x+1)^{2}}, \quad \cdots$

and

$$
E_{0}=1, \quad E_{1}=-\frac{1}{4}, \quad E_{2}=-\frac{9}{64}, \quad \cdots .
$$

Both expansions $S=S_{0}+g^{-1} S_{1}+g^{-2} S_{2}+\cdots$ and $E=g E_{0}+E_{1}+g^{-1} E_{2}+\cdots$ are divergent, furthermore, at $x=-1$ and for $n \geq 1$, each $S_{n}(x)$ is infinite. The reflection $x \rightarrow-x$ gives a corresponding asymptotic expansion $S_{n}(x) \rightarrow$ $S_{n}(-x)$, in which each $S_{n}(-x)$ is regular at $x=-1$, but singular at $x=+1$.

As noted in section 1 , for $g$ large, the first few terms of the perturbative series (with (A.31) for $x$ positive and the corresponding expansion $S_{n}(x) \rightarrow S_{n}(-x)$ for $x$ negative) give a fairly good description of the true wave function $\psi(x)$ whenever $\psi(x)$ is large (i.e. for $x$ near \pm 1 ). However, for $x$ near zero, when $\psi(x)$ is exponentially small, the perturbative series becomes totally unreliable. This suggests the use of first few terms of the perturbative series for regions whenever $\psi(x)$ is expected to be large. In regions where $\psi(x)$ is exponentially small, simple interpolations by hand may already be adequate for a trial function, as we shall see. Since the quartic potential (A.29) is even in $x$, so is the groundstate wave function; likewise, we require the trial function $\phi(x)$ also to satisfy $\phi(x)=\phi(-x)$. At $x=0$, we require

$$
\left(\frac{d \phi}{d x}\right)_{x=0}=0 .
$$

To construct $\phi(x)$, we start with the first two functions $S_{0}(x)$ and $S_{1}(x)$ in (A.31). Introduce, for $x \geq 0$,

$$
\phi_{+}(x) \equiv e^{-g S_{0}(x)-S_{1}(x)}=\left(\frac{2}{1+x}\right) e^{-g S_{0}(x)}
$$

and

$$
\phi_{-}(x) \equiv e^{-g S_{0}(-x)-S_{1}(x)}=\left(\frac{2}{1+x}\right) e^{-\frac{4}{3} g+g S_{0}(x)} .
$$


In order to satisfy (A.33), we define

$$
\phi(x)=\phi(-x) \equiv \begin{cases}\phi_{+}(x)+\frac{g-1}{g+1} \phi_{-}(x), & \text { for } \quad 0 \leq x<1 \\ \left(1+\frac{g-1}{g+1} e^{-\frac{4}{3} g}\right) \phi_{+}(x), & \text { for } \quad x>1\end{cases}
$$

Thus, by construction (A.33) is satisfied. Furthermore, $\phi(x)$ is continuous everywhere, for $x$ from $-\infty$ to $\infty$, and so is its derivative.

By differentiating $\phi_{+}(x)$ and $\phi(x)$, we see that they satisfy

$$
(T+V+u) \phi_{+}=g \phi_{+}
$$

and

$$
(T+V+h) \phi=g \phi,
$$

where

$$
u(x)=\frac{1}{(1+x)^{2}}
$$

and

$$
h(x)=h(-x)
$$

with, for $x \geq 0$

$$
h(x)=u(x)+\hat{g}(x)
$$

where

$$
\hat{g}(x)= \begin{cases}2 g \frac{(g-1) e^{2 g S_{0}(x)-\frac{4}{3} g}}{(g+1)+(g-1) e^{2 g S_{0}(x)-\frac{4}{3} g}}, & \text { for } 0 \leq x<1 \\ 0 & \text { for } x>1 .\end{cases}
$$

Note that for $g>1, \hat{g}(x)$ is positive, and has a discontinuity at $x=1$. Furthermore, for $x$ positive both $u(x)$ and $\hat{g}(x)$ are decreasing functions of $x$. Therefore, $h(x)$ also satisfies (4.4) for $x=r>0$. 


\section{Appendix B. Proof of the Hierarchy Theorem}

In this Appendix, we give the proof of the Hierarchy Theorem stated in Section 4 . The discussion follows very closely the one given for the one-dimensional case in Ref. 19. We first establish several lemmas applicable to both boundary conditions (4.6) and (4.7): $(A) f_{n}(\infty)=1$ and $(B) f_{n}(0)=1$.

Lemma 1 For any pair $f_{m}(r)$ and $f_{l}(r)$

i) if at all $r, \frac{d}{d r}\left(\frac{f_{m}(r)}{f_{l}(r)}\right)<0 \quad$ then $\quad \mathcal{E}_{m+1}>\mathcal{E}_{l+1}$,

and

ii) if at all $r, \frac{d}{d r}\left(\frac{f_{m}(r)}{f_{l}(r)}\right)>0 \quad$ then $\quad \mathcal{E}_{m+1}<\mathcal{E}_{l+1}$.

Proof

According to (4.9)

$$
\mathcal{E}_{m+1}\left[f_{m}\right]=\left[h f_{m}\right]
$$

Also by definition (4.10),

$$
\mathcal{E}_{l+1}\left[f_{m}\right]=\left[\mathcal{E}_{l+1} f_{m}\right]
$$

Their difference gives

$$
\left(\mathcal{E}_{m+1}-\mathcal{E}_{l+1}\right)\left[f_{m}\right]=\left[\left(h-\mathcal{E}_{l+1}\right) f_{m}\right] .
$$

From (B.3),

$$
0=\left[\left(h-\mathcal{E}_{l+1}\right) f_{l}\right] .
$$

Let $r_{l+1}$ be defined by (4.21); i.e., at $r=r_{l+1}$,

$$
h\left(r_{l+1}\right)=\mathcal{E}_{l+1} .
$$

Multiplying (B.5) by $f_{l}\left(r_{l+1}\right)$ and (B.6) by $f_{m}\left(r_{l+1}\right)$ and taking their difference, we have

$$
f_{l}\left(r_{l+1}\right)\left(\mathcal{E}_{m+1}-\mathcal{E}_{l+1}\right)\left[f_{m}\right]=\left[\left(h-\mathcal{E}_{l+1}\right)\left(f_{m}(r) f_{l}\left(r_{l+1}\right)-f_{l}(r) f_{m}\left(r_{l+1}\right)\right)\right],
$$


in which the unsubscripted $r$ acts as a dummy variable; thus $\left[f_{m}(r)\right]$ means $\left[f_{m}\right]$ and $\left[f_{m}\left(r_{l+1}\right)\right]$ means $f_{m}\left(r_{l+1}\right) \cdot[1]$, etc.

(i) If $\left(f_{m}(r) / f_{l}(r)\right)^{\prime}<0$, then for $r<r_{l+1}$

$$
\frac{f_{m}(r)}{f_{l}(r)}>\frac{f_{m}\left(r_{l+1}\right)}{f_{l}\left(r_{l+1}\right)}
$$

In addition, since in accordance with (4.4) and (B.7), $h^{\prime}(r)<0$ and $h\left(r_{l+1}\right)=$ $\mathcal{E}_{l+1}$, we also have for $r<r_{l+1}$

$$
h(r)>\mathcal{E}_{l+1} .
$$

Thus, the function inside the square bracket on the right hand side of (B.8) is positive for $r<r_{l+1}$. Also, the inequalities (B.9) and (B.10) both reverse their signs for $r>r_{l+1}$. Consequently, the right hand side of (B.8) is positive definite, and so is its left side. Therefore, on account of (4.25A)-(4.25B), (B.1) holds.

(ii) If $\left(f_{m}(r) / f_{l}(r)\right)^{\prime}>0$, we see that for $r<r_{l+1}$, (B.9) reverses its sign but not (B.10). A similar reversal of sign happens for $r>r_{l+1}$. Thus, the right hand side of (B.8) is now negative definite and therefore $\mathcal{E}_{m+1}<\mathcal{E}_{l+1}$. Lemma 1 is proved.

Let

$$
\eta=\eta(\xi)
$$

be a single valued differentiable function of $\xi$ in the range between $a$ and $b$ with

$$
0 \leq a \leq \xi \leq b
$$

and with

$$
\eta(a) \geq 0
$$

Lemma 2.

(i) The ratio $\eta / \xi$ is a decreasing function of $\xi$ for $a<\xi<b$ if

$$
\frac{d \eta}{d \xi} \leq \frac{\eta}{\xi} \quad \text { at } \quad \xi=a
$$

and

$$
\frac{d^{2} \eta}{d \xi^{2}}<0 \quad \text { for } \quad a<\xi<b .
$$


(ii) The ratio $\eta / \xi$ is an increasing function of $\xi$ for $a<\xi<b$ if

$$
\frac{d \eta}{d \xi} \geq \frac{\eta}{\xi} \quad \text { at } \quad \xi=a
$$

and

$$
\frac{d^{2} \eta}{d \xi^{2}}>0 \quad \text { for } \quad a<\xi<b
$$

Proof Define

$$
L \equiv \xi \frac{d \eta}{d \xi}-\eta
$$

to be the Legendre transform $L(\xi)$. We have

$$
\frac{d L}{d \xi}=\xi \frac{d^{2} \eta}{d \xi^{2}}
$$

and

$$
\frac{d}{d \xi}\left(\frac{\eta}{\xi}\right)=\frac{L}{\xi^{2}}
$$

Since (B.14) says that $L(a) \leq 0$ and (B.15) says that $\frac{d L}{d \xi}<0$ for $a<\xi<b$, these two conditions imply $L(\xi)<0$ for $a<\xi<b$, which proves (i) in view of (B.20). The proof of (ii) is the same, but with inequalities reversed.

Lemma 3 For any pair $f_{m}(r)$ and $f_{l}(r)$

(i) if over all $r$,

$$
\frac{d}{d r}\left(\frac{f_{m}(r)}{f_{l}(r)}\right)<0 \quad \text { then at all } r, \quad \frac{d}{d r}\left(\frac{\mathcal{D}_{m+1}(r)}{\mathcal{D}_{l+1}(r)}\right)<0,
$$

and (ii) if over all $r$,

$$
\frac{d}{d r}\left(\frac{f_{m}(r)}{f_{l}(r)}\right)>0 \quad \text { then at all } r, \quad \frac{d}{d r}\left(\frac{\mathcal{D}_{m+1}(r)}{\mathcal{D}_{l+1}(r)}\right)>0 .
$$

Proof From (4.18) and (4.20), we have

$$
\mathcal{D}_{m+1}^{\prime}(r)=\left(h(r)-\mathcal{E}_{m+1}\right) \chi^{2}(r) f_{m}(r)
$$

and

$$
\mathcal{D}_{l+1}^{\prime}(r)=\left(h(r)-\mathcal{E}_{l+1}\right) \chi^{2}(r) f_{l}(r) .
$$


Define

$$
\xi=\mathcal{D}_{l+1}(r) \text { and } \eta=\mathcal{D}_{m+1}(r) .
$$

In any local region of $r$ where $\mathcal{D}_{l+1}^{\prime}(r) \neq 0$, we can regard $\eta=\eta(\xi)$ through $\eta(r)=\eta(\xi(r))$. Hence, we have

$$
\frac{d \eta}{d \xi}=\frac{\mathcal{D}_{m+1}^{\prime}(r)}{\mathcal{D}_{l+1}^{\prime}(r)}=R(r) \frac{f_{m}(r)}{f_{l}(r)}
$$

where

$$
R(r)=\frac{h(r)-\mathcal{E}_{m+1}}{h(r)-\mathcal{E}_{l+1}}
$$

and

$$
\begin{aligned}
\frac{d}{d \xi}\left(\frac{d \eta}{d \xi}\right) & =\frac{1}{\mathcal{D}_{l+1}^{\prime}}\left(\frac{\mathcal{D}_{m+1}^{\prime}}{\mathcal{D}_{l+1}^{\prime}}\right)^{\prime}=\frac{1}{\mathcal{D}_{l+1}^{\prime}}\left(R \frac{f_{m}}{f_{l}}\right)^{\prime} \\
& =\frac{1}{\mathcal{D}_{l+1}^{\prime}}\left(R^{\prime} \frac{f_{m}}{f_{l}}+R\left(\frac{f_{m}}{f_{l}}\right)^{\prime}\right)
\end{aligned}
$$

where

$$
R^{\prime}(r)=\frac{\mathcal{E}_{m+1}-\mathcal{E}_{l+1}}{\left(h(r)-\mathcal{E}_{l+1}\right)^{2}} h^{\prime}(r)
$$

(i) If $\left(f_{m} / f_{l}\right)^{\prime}<0$, from Lemma 1 , we have

$$
\mathcal{E}_{m+1}>\mathcal{E}_{l+1}
$$

From $h^{\prime}(r)<0$ and the definition of $r_{m+1}$ and $r_{l+1}$, given by (B.7), we have

$$
\begin{gathered}
r_{m+1}<r_{l+1}, \\
h\left(r_{m+1}\right)=\mathcal{E}_{m+1} \quad \text { and } \quad h\left(r_{l+1}\right)=\mathcal{E}_{l+1} .
\end{gathered}
$$

We note that from (4.18) and (4.20) $\mathcal{D}_{m+1}(r)$ and $\mathcal{D}_{l+1}(r)$ are both positive continuous functions of $r$, varying from at $r=0$,

$$
\mathcal{D}_{m+1}(0)=\mathcal{D}_{l+1}(0)=0
$$

to at $r=\infty$

$$
\mathcal{D}_{m+1}(\infty)=\mathcal{D}_{l+1}(\infty)=0
$$


with their maxima at $r_{m+1}$ for $\mathcal{D}_{m+1}(r)$ and $r_{l+1}$ for $\mathcal{D}_{l+1}(r)$, since in accordance with (B.23)-(B.24) and (B.32),

$$
\mathcal{D}_{m+1}^{\prime}\left(r_{m+1}\right)=0 \quad \text { and } \quad \mathcal{D}_{l+1}^{\prime}\left(r_{l+1}\right)=0 .
$$

From (B.29)-(B.30), we see that $R^{\prime}(r)$ is always $<0$. Furthermore, from (B.27), we also find that the function $R(r)$ has a discontinuity at $r=r_{l+1}$. At $r=0, R(0)$ satisfies

$$
0<R(0)=\frac{h(0)-\mathcal{E}_{m+1}}{h(0)-\mathcal{E}_{l+1}}<1 .
$$

As $r$ increases from $0, R(r)$ decreases from $R(0)$, through

$$
R\left(r_{m+1}\right)=0,
$$

to $-\infty$ at $r=r_{l+1}-; R(r)$ then switches to $+\infty$ at $r=r_{l+1}+$, and continues to decrease as $r$ increases from $r_{l+1}+$. At $r=\infty, R(r)$ becomes

$$
R(\infty)=\frac{\mathcal{E}_{m+1}}{\mathcal{E}_{l+1}}>1
$$

It is convenient to divide the $r$-axis into three regions:

$$
\begin{array}{lr}
\text { (I) } & 0<r<r_{m+1}, \\
\text { (II) } & r_{m+1}<r<r_{l+1} \\
\text { (III) } & r_{l+1}<r .
\end{array}
$$

The signs of $\mathcal{D}_{m+1}^{\prime}, \mathcal{D}_{l+1}^{\prime}, R$ and $R^{\prime}$ in these regions are summarized in Table 1 . Assuming $\left(f_{m} / f_{l}\right)^{\prime}<0$ we shall show the validity of (B.21), $\left(\mathcal{D}_{m+1} / \mathcal{D}_{l+1}\right)^{\prime}<0$, in each of these three regions.

Table 1. The signs of $\mathcal{D}_{m+1}^{\prime}(r), \mathcal{D}_{l+1}^{\prime}(r), h(r)-\mathcal{E}_{m+1}, h(r)-\mathcal{E}_{l+1}$, $R(r)$ and $R^{\prime}(r)$ in the three regions defined by (B.39), when $\mathcal{E}_{m+1}>\mathcal{E}_{l+1}$.

\begin{tabular}{|c|c|c|c|c|c|c|}
\hline region & $\mathcal{D}_{m+1}^{\prime}(r)$ & $\mathcal{D}_{l+1}^{\prime}(r)$ & $h(r)-\mathcal{E}_{m+1}$ & $h(r)-\mathcal{E}_{l+1}$ & $R(r)$ & $R^{\prime}(r)$ \\
\hline I & $>0$ & $>0$ & $>0$ & $>0$ & $>0$ & $<0$ \\
II & $<0$ & $>0$ & $<0$ & $>0$ & $<0$ & $<0$ \\
III & $<0$ & $<0$ & $<0$ & $<0$ & $>0$ & $<0$ \\
\hline
\end{tabular}


Since

$$
\mathcal{E}_{l+1}<h(r)<\mathcal{E}_{m+1} \quad \text { in } \quad \text { II, }
$$

$\mathcal{D}_{m+1}(r)$ is decreasing and $\mathcal{D}_{l+1}(r)$ is increasing; it is clear that (B.21) holds in II.

In each of regions (I) and (III), we have $R(r)>0$ from (B.27) and $R^{\prime}(r)<$ 0 from (B.29). Since $\left(f_{m} / f_{l}\right)^{\prime}$ is always negative by the assumption in (B.21), both terms inside the big parenthesis of (B.28) are negative; hence the same (B.28) states that $d^{2} \eta / d \xi^{2}$ has the opposite sign from $\mathcal{D}_{l+1}^{\prime}$. From the sign of $\mathcal{D}_{l+1}^{\prime}$ listed in Table 1 , we see that

$$
\frac{d^{2} \eta}{d \xi^{2}}<0 \quad \text { in }
$$

and

$$
\frac{d^{2} \eta}{d \xi^{2}}>0 \quad \text { in } \quad \text { (III). }
$$

Within each region, $\eta=\mathcal{D}_{m+1}(r)$ and $\xi=\mathcal{D}_{l+1}(r)$ are both monotonic in $r$; therefore, $\eta$ is a single-valued function of $\xi$ and we can apply Lemma 2. In (I), at $r=0$, both $\mathcal{D}_{m+1}(0)$ and $\mathcal{D}_{l+1}(0)$ are 0 according to (4.20), but their ratio is given by

$$
\frac{\mathcal{D}_{m+1}(0)}{\mathcal{D}_{l+1}(0)}=\frac{\mathcal{D}_{m+1}^{\prime}(0)}{\mathcal{D}_{l+1}^{\prime}(0)}
$$

Therefore,

$$
\left(\frac{d \eta}{d \xi}\right)_{r=0}=\left(\frac{\eta}{\xi}\right)_{r=0} .
$$

Furthermore, from (B.41), $\frac{d^{2} \eta}{d \xi^{2}}<0$ in (I), it follows from Lemma 2, case(i), the ratio $\eta / \xi$ is a decreasing function of $\xi$. Since $\xi^{\prime}=\mathcal{D}_{l+1}^{\prime}$ is $>0$ in (I) according to (B.24), we have

$$
\frac{d}{d r}\left(\frac{\mathcal{D}_{m+1}}{\mathcal{D}_{l+1}}\right)<0 \quad \text { in } \quad(\mathrm{I}) .
$$

In (III), at $r=\infty$, both $\mathcal{D}_{m+1}(\infty)$ and $\mathcal{D}_{l+1}(\infty)$ are 0 according to (B.34). Their ratio is

$$
\frac{\mathcal{D}_{m+1}(\infty)}{\mathcal{D}_{l+1}(\infty)}=\frac{\mathcal{D}_{m+1}^{\prime}(\infty)}{\mathcal{D}_{l+1}^{\prime}(\infty)}
$$


which gives at $r=\infty$,

$$
\left(\frac{d \eta}{d \xi}\right)_{r=\infty}=\left(\frac{\eta}{\xi}\right)_{r=\infty}
$$

As $r$ decreases from $r=\infty$ to $r=r_{l+1}$, from (B.42) we have $\frac{d^{2} \eta}{d \xi^{2}}>0$ in (III). It follows from Lemma 2, case (ii), $\eta / \xi$ is an increasing function of $\xi$. Since $\xi^{\prime}=\mathcal{D}_{l+1}^{\prime}$ is $<0$, because $r>r_{l+1}$, we have

$$
\frac{d}{d r}\left(\frac{\mathcal{D}_{m+1}}{\mathcal{D}_{l+1}}\right)<0 \quad \text { in } \quad(\text { III }) .
$$

Thus, we prove case(i) of Lemma 3. Case(ii) of Lemma 3 follows from case (i) through the interchange of the subscripts $m$ and $l$. Lemma 3 is then established.

Lemma 4 Take any pair $f_{m}(r)$ and $f_{l}(r)$

(A) For the boundary condition $f_{n}(\infty)=1$, if at all $r$,

$$
\frac{d}{d r}\left(\frac{f_{m}(r)}{f_{l}(r)}\right)<0 \text { then at all } r, \frac{d}{d r}\left(\frac{f_{m+1}(r)}{f_{l+1}(r)}\right)<0 ;
$$

therefore, if at all $r$,

$$
\frac{d}{d r}\left(\frac{f_{m}(r)}{f_{l}(r)}\right)>0 \text { then at all } r, \frac{d}{d r}\left(\frac{f_{m+1}(r)}{f_{l+1}(r)}\right)>0 .
$$

$(B)$ For the boundary condition $f_{n}(0)=1$, if at all $r$,

$$
\frac{d}{d r}\left(\frac{f_{m}(r)}{f_{l}(r)}\right)<0 \text { then at all } r, \frac{d}{d r}\left(\frac{f_{m+1}(r)}{f_{l+1}(r)}\right)>0 ;
$$

therefore, if at all $r$,

$$
\frac{d}{d r}\left(\frac{f_{m}(r)}{f_{l}(r)}\right)>0 \text { then at all } r, \frac{d}{d r}\left(\frac{f_{m+1}(r)}{f_{l+1}(r)}\right)<0 .
$$

Proof Define

$$
\hat{\xi}=f_{l+1}(r) \quad \text { and } \quad \hat{\eta}=f_{m+1}(r) .
$$

From (4.15) we see that

$$
\frac{d \hat{\eta}}{d \hat{\xi}}=\frac{f_{m+1}^{\prime}(r)}{f_{l+1}^{\prime}(r)}=\frac{\mathcal{D}_{m+1}(r)}{\mathcal{D}_{l+1}(r)}
$$


and

$$
\frac{d}{d \hat{\xi}}\left(\frac{d \hat{\eta}}{d \hat{\xi}}\right)=\frac{1}{f_{l+1}^{\prime}} \frac{d}{d r}\left(\frac{\mathcal{D}_{m+1}(r)}{\mathcal{D}_{l+1}(r)}\right) .
$$

(A) In this case $f_{n}(\infty)=1$ for all $n$. Thus, at $r=\infty, \hat{\xi}=f_{l+1}(\infty)=1$, $\hat{\eta}=f_{m+1}(\infty)=1$, and their ratio

$$
\left(\frac{\hat{\eta}}{\hat{\xi}}\right)_{r=\infty}=1 .
$$

At the same point $r=\infty$, in accordance with (4.18), $\mathcal{D}_{l+1}(\infty)=\mathcal{D}_{m+1}(\infty)=$ 0 , but their ratio is, on account of $h(\infty)=0$ and (B.1) of Lemma 1 ,

$$
\frac{\mathcal{D}_{m+1}(\infty)}{\mathcal{D}_{l+1}(\infty)}=\frac{\mathcal{D}_{m+1}^{\prime}(\infty)}{\mathcal{D}_{l+1}^{\prime}(\infty)}=\frac{h(\infty)-\mathcal{E}_{m+1}}{h(\infty)-\mathcal{E}_{l+1}}=\frac{\mathcal{E}_{m+1}}{\mathcal{E}_{l+1}}>1
$$

in which the last inequality follows from the same assumption, if $\left(f_{m} / f_{l}\right)^{\prime}<0$, shared by (B.1) of Lemma 1 and the present (B.49A) that we wish to prove. Thus, from (B.52), at $r=\infty$

$$
\left(\frac{d \hat{\eta}}{d \hat{\xi}}\right)_{r=\infty}=\frac{\mathcal{E}_{m+1}}{\mathcal{E}_{l+1}}>\left(\frac{\hat{\eta}}{\hat{\xi}}\right)_{r=\infty}=1 .
$$

As $r$ decreases from $\infty$ to $0, \hat{\xi}$ increases from $f_{l+1}(\infty)=1$ to $f_{l+1}(0)>1$, in accordance with (4.24) and (4.25A). On account of (B.21) of Lemma 3, we have $\left(\mathcal{D}_{m+1} / \mathcal{D}_{l+1}\right)^{\prime}<0$, which when combined with (B.53) and $f_{n}^{\prime}(r)<0$ leads to

$$
\frac{d}{d \hat{\xi}}\left(\frac{d \hat{\eta}}{d \hat{\xi}}\right)>0
$$

Thus, by using (B.16)-(B.17) of Lemma 2 , we have $\hat{\eta} / \hat{\xi}$ to be an increasing function of $\hat{\xi}$; i.e.,

$$
\frac{d}{d \hat{\xi}}\left(\frac{\hat{\eta}}{\hat{\xi}}\right)>0
$$

Because

$$
\frac{d}{d r}\left(\frac{\hat{\eta}}{\hat{\xi}}\right)=\hat{\xi}^{\prime} \frac{d}{d \hat{\xi}}\left(\frac{\hat{\eta}}{\hat{\xi}}\right)=f_{l+1}^{\prime} \frac{d}{d \hat{\xi}}\left(\frac{\hat{\eta}}{\hat{\xi}}\right)
$$


and $f_{l+1}^{\prime}<0$, we find

$$
\frac{d}{d r}\left(\frac{f_{m+1}}{f_{l+1}}\right)=\frac{d}{d r}\left(\frac{\hat{\eta}}{\hat{\xi}}\right)<0,
$$

which establishes (B.49A). Through the interchange of the subscripts $m$ and $l$, we also obtain (B.50A).

Next, we turn to Case $(B)$ with the boundary condition $f_{n}(0)=1$ for all $n$. Therefore at $r=0$,

$$
\frac{f_{m+1}(0)}{f_{l+1}(0)}=1
$$

Furthermore from (4.15) and (4.16B), we also have $f_{m+1}^{\prime}(0)=f_{l+1}^{\prime}(0)=0$ and $\mathcal{D}_{m+1}(0)=\mathcal{D}_{l+1}(0)=0$, with their ratio given by

$$
\begin{aligned}
\left(\frac{d f_{m+1}}{d f_{l+1}}\right)_{r=0} & =\frac{f_{m+1}^{\prime}(0)}{f_{l+1}^{\prime}(0)}=\frac{\mathcal{D}_{m+1}(0)}{\mathcal{D}_{l+1}(0)}=\frac{\mathcal{D}_{m+1}^{\prime}(0)}{\mathcal{D}_{l+1}^{\prime}(0)} \\
& =\frac{h(0)-\mathcal{E}_{m+1}}{h(0)-\mathcal{E}_{l+1}} .
\end{aligned}
$$

From (B.1) of Lemma 1, we see that if $\left(f_{m} / f_{l}\right)^{\prime}<0$, then $\mathcal{E}_{m+1}>\mathcal{E}_{l+1}$ and therefore

Thus,

$$
\begin{gathered}
\left(\frac{d f_{m+1}}{d f_{l+1}}\right)_{r=0}<1, \\
\left(\frac{\hat{\eta}}{\hat{\xi}}\right)_{r=0}=1 .
\end{gathered}
$$

$$
\left(\frac{d \hat{\eta}}{d \hat{\xi}}\right)_{r=0}<\left(\frac{\hat{\eta}}{\hat{\xi}}\right)_{r=0} .
$$

Analogously to (B.18), define

$$
L(r) \equiv \hat{\xi} \frac{d \hat{\eta}}{d \hat{\xi}}-\hat{\eta}=f_{l+1}(r) \frac{f_{m+1}^{\prime}(r)}{f_{l+1}^{\prime}(r)}-f_{m+1}(r) ;
$$

therefore

$$
\begin{aligned}
\frac{d L(r)}{d r} & =\hat{\xi}^{\prime} \frac{d L}{d \hat{\xi}}=\hat{\xi}^{\prime} \hat{\xi} \frac{d}{d \hat{\xi}} \frac{d \hat{\eta}}{d \hat{\xi}} \\
& =\hat{\xi} \frac{d}{d r}\left(\frac{d \hat{\eta}}{d \hat{\xi}}\right)=f_{l+1} \frac{d}{d r}\left(\frac{f_{m+1}^{\prime}}{f_{l+1}^{\prime}}\right)=f_{l+1} \frac{d}{d r}\left(\frac{\mathcal{D}_{m+1}}{\mathcal{D}_{l+1}}\right)
\end{aligned}
$$


From (B.21) of Lemma 3, we know that if $\left(f_{m} / f_{l}\right)^{\prime}<0$ then $\left(\mathcal{D}_{m+1} / \mathcal{D}_{l+1}\right)^{\prime}<$ 0 , which leads to

$$
\frac{d L(r)}{d r}<0 .
$$

From (B.66), we have

$$
L(r)=\hat{\xi}\left(\frac{d \hat{\eta}}{d \hat{\xi}}-\frac{\hat{\eta}}{\hat{\xi}}\right)=f_{l+1}(r)\left(\frac{d \hat{\eta}}{d \hat{\xi}}-\frac{\hat{\eta}}{\hat{\xi}}\right),
$$

and therefore at $r=0$, because of (B.65),

$$
L(0)<0 .
$$

Combining (B.68) and (B.70), we derive

$$
L(r)<0 \quad \text { for } \quad r \geq 0 .
$$

Multiplying (B.66) by $f_{l+1}^{\prime}(r)$, we have

$$
\begin{aligned}
f_{l+1}^{\prime}(r) L(r) & =f_{l+1}(r) f_{m+1}^{\prime}(r)-f_{m+1}(r) f_{l+1}^{\prime}(r) \\
& =f_{l+1}^{2}(r)\left(\frac{f_{m+1}(r)}{f_{l+1}(r)}\right)^{\prime} .
\end{aligned}
$$

Because $f_{l+1}^{\prime}(r)$ and $L(r)$ are both negative, it follows then

$$
\left(\frac{f_{m+1}(r)}{f_{l+1}(r)}\right)^{\prime}>0
$$

which gives (B.49B) for Case (B), with the boundary condition $f_{n}(0)=1$. Interchanging the subscripts $m$ and $l$, (B.49B) becomes (B.50B), and Lemma 4 is established.

We now turn to the proof of the theorem stated in (4.26)-(4.34).

Proof of the Hierarchy Theorem

When $n=0$, we have

$$
f_{0}(r)=1 .
$$

From (4.22)-(4.24), we find for $n=1$

$$
f_{1}^{\prime}(r)<0,
$$


and therefore

$$
\left(f_{1} / f_{0}\right)^{\prime}<0 .
$$

In Case $(A)$, by using (B.49A) and by setting $m=1$ and $l=0$, we derive $\left(f_{2} / f_{1}\right)^{\prime}<0$; through induction, it follows then $\left(f_{n+1} / f_{n}\right)^{\prime}<0$ for all $n$. From Lemma 1 , we also find $\mathcal{E}_{n+1}>\mathcal{E}_{n}$ for all n. Thus, (4.26)-(4.29) are established.

In Case (B), by using (B.75) and (B.49B), and setting $m=1$ and $l=0$, we find $\left(f_{2} / f_{1}\right)^{\prime}>0$, which in turn leads to $\left(f_{3} / f_{2}\right)^{\prime}<0, \cdots$, and (4.33)(4.34). Inequalities (4.30)-(4.32) now follow from (B.1)-(B.2) of Lemma 1. The Hierarchy Theorem is proved.

Assuming that $h(0)$ is finite, we have for any $n$

$$
0<\mathcal{E}_{n}<h(0) \text {. }
$$

Therefore, each of the monotonic sequences

$$
\begin{aligned}
& \mathcal{E}_{1}<\mathcal{E}_{2}<\mathcal{E}_{3}<\cdots, \quad \text { in }(A) \\
& \mathcal{E}_{1}<\mathcal{E}_{3}<\mathcal{E}_{5}<\cdots, \quad \text { in } \quad(B)
\end{aligned}
$$

and

$$
\mathcal{E}_{2}>\mathcal{E}_{4}>\mathcal{E}_{6}>\cdots, \quad \text { in }(B)
$$

converges to a finite limit $\mathcal{E}$. By following the discussions in Section 5 of Ref.[18], one can show that each of the corresponding monotonic sequences of $f_{n}(r)$ also converges to a finite limit $f(r)$. The interchange of the limit $n \rightarrow \infty$ and the integrations in (4.16A) completes the proof that in Case (A) the limits $\mathcal{E}$ and $f(r)$ satisfy

$$
f(r)=1-2 \int_{r}^{\infty} \chi^{-2}(y) d y \int_{y}^{\infty} \chi^{2}(z)(h(z)-\mathcal{E}) f(z) d z .
$$

As noted before, the convergence in Case $(A)$ can hold for any large but finite and positive $h(r)$, provided that $h^{\prime}(r)$ is negative for $r>0$. In Case (B), a large $h(r)$ may yield a negative $f_{n}(r)$, in violation of (4.25B) . Therefore, the convergence does depend on the smallness of $h(r)$. One has to follow discussions similar to those given in Ref.[17] to ensure that the limits $\mathcal{E}$ and $f(r)$ satisfy

$$
f(r)=1-2 \int_{0}^{r} \chi^{-2}(y) d y \int_{0}^{y} \chi^{2}(z)(h(z)-\mathcal{E}) f(z) d z .
$$




\section{Appendix C. Comparison with Perturbative Expansion}

\section{C.1 A simple Model and Its Iterative Solutions}

Consider the one-dimensional problem in which $V, U$ and $h$ of (2.1), (2.7) and (2.11) are given by

$$
\begin{aligned}
& V(x)=V(-x)=\left\{\begin{array}{lr}
\infty & |x|>L+l \\
0 & \text { for } \quad l<|x|<L+l, \\
-\frac{1}{2} g^{2} & \\
& |x|<l
\end{array}\right. \\
& U(x)=U(-x)=\left\{\begin{array}{lll}
\infty & & |x|>L+l, \\
0 & \text { for } & |x|<L+l
\end{array}\right.
\end{aligned}
$$

and

$$
h(x)=U(x)-V(x)=\left\{\begin{array}{lll}
0 & |x|>l, \\
\frac{1}{2} g^{2} & \text { for } & |x|<l .
\end{array}\right.
$$

Let $\phi(x)$ and $\psi(x)$ be the respective groundstates of

$$
H_{0} \phi=-\frac{1}{2} \frac{d^{2} \phi}{d x^{2}}+U \phi=E_{0} \phi
$$

and

$$
H \psi=-\frac{1}{2} \frac{d^{2} \psi}{d x^{2}}+V \psi=E \psi .
$$

As a model, (C.5) is the Schroedinger equation that we wish to solve, and (C.4) is the equation that the trial function $\phi$ satisfies. The iterative equation (2.19) becomes

$$
\left(-\frac{1}{2} \frac{d^{2}}{d x^{2}}+U(x)-E_{0}\right) \psi_{n}(x)=\left(h(x)-\mathcal{E}_{n}\right) \psi_{n-1}(x) .
$$

with $\psi_{0}=\phi(x)$. Since $U$ and $V$ are both even in $x$, we need only to consider the region

$$
x \geq 0 \text {. }
$$


Set within $x<L+l$

$$
\phi(x)=\cos p x
$$

with

$$
p(L+l)=\frac{\pi}{2} \quad \text { and } \quad E_{0}=\frac{p^{2}}{2} .
$$

Introduce, as in (2.25), $f_{n}=\psi_{n} / \phi$. We have in accordance with (3.1)-(3.7)

$$
\begin{gathered}
D_{n}(x)=-\frac{1}{2} \kappa(x) \frac{d f_{n}}{d x}, \quad \kappa(x)=\phi^{2}(x), \\
\frac{d D_{n}}{d x}=\sigma_{n}(x)=\left(h(x)-\mathcal{E}_{n}\right) \phi^{2}(x) f_{n-1}(x), \\
\int_{0}^{\infty} \sigma_{n}(x) d x=0
\end{gathered}
$$

and therefore

$$
\mathcal{E}_{n}=\frac{\int_{0}^{\infty} h \phi^{2} f_{n-1} d x}{\int_{0}^{\infty} \phi^{2} f_{n-1} d x}
$$

One can verify that for $n=1$

$$
\begin{gathered}
\mathcal{E}_{1}=\frac{g^{2}}{2(L+l)}\left(l+\frac{1}{2 p} \sin 2 p l\right), \\
D_{1}(x)=\frac{g^{2}}{4(L+l)} \begin{cases}\left(l+\frac{\sin 2 p l}{2 p}\right)\left(L+l-x-\frac{\sin 2 p x}{2 p}\right), & l<x<L+l, \\
\left(x+\frac{\sin 2 p x}{2 p}\right)\left(L-\frac{\sin 2 p l}{2 p}\right), & 0<x<l\end{cases}
\end{gathered}
$$

and

$f_{1}(x)= \begin{cases}f_{1}(R)+\frac{\mathcal{E}_{1}}{p^{2}}[1-p(L+l-x) \tan p x], & l<x<L+l, \\ f_{1}(0)-\frac{1}{p}\left(\frac{g^{2}}{2}-\mathcal{E}_{1}\right) x \tan p x, & 0<x<l .\end{cases}$

The constants $f_{1}(R)$ and $f_{1}(0)$ are related through the continuity of $f_{1}(x)$ at $x=l$. In order to derive the general solution for $f_{n}(x)$, it is useful to consider a limited region in $x$ (either $|x|<l$ or $|x|>l$ ) over which

$$
\Delta_{n} \equiv 2\left(h(x)-\mathcal{E}_{n}\right)=\text { constant }
$$


From (C.10)-(C.11), we have

$$
-\frac{d}{d x}\left(\cos ^{2} p x \frac{d f_{n}}{d x}\right)=\Delta_{n} \cos ^{2} p x f_{n-1} .
$$

Write

$$
f_{n}=P_{n}(x)+(\tan p x) Q_{n}(x) .
$$

We find, from (C.18),

$$
\frac{d^{2}}{d x^{2}} P_{n}+2 p \frac{d}{d x} Q_{n}=-\Delta_{n} P_{n-1}
$$

and

$$
\frac{d^{2}}{d x^{2}} Q_{n}-2 p \frac{d}{d x} P_{n}=-\Delta_{n} Q_{n-1} .
$$

Thus, $P_{n}$ and $Q_{n}$ are both $n^{\text {th }}$ order polynomials in $x$. Define

$$
P_{n}^{ \pm} \equiv \frac{1}{2}\left(P_{n} \mp i Q_{n}\right)=\left(P_{n}^{\mp}\right)^{*} .
$$

From (C.20), $P_{n}^{ \pm}$satisfies

$$
\left(\frac{d^{2}}{d x^{2}} \pm 2 i p \frac{d}{d x}\right) P_{n}^{ \pm}=-\Delta_{n} P_{n-1}^{ \pm} .
$$

It is useful to introduce a set of $n^{\text {th }}$ order polynomials $R_{n}^{ \pm}$, independent of $\Delta_{n}$. We require

$$
\left(\frac{d^{2}}{d x^{2}} \pm 2 i p \frac{d}{d x}\right) R_{n}^{ \pm}=-R_{n-1}^{ \pm}
$$

with the boundary conditions (i) for $n=0$,

$$
R_{0}^{ \pm}(x)=1 \quad \text { at all } x,
$$

and (ii) for $n>0$, at $x=0$

$$
R_{n}^{ \pm}(0)=0 .
$$

The solution for $R_{n}^{ \pm}(x)$ can be further simplified by introducing a set of $n^{\text {th }}$ order polynomials $R_{n}(x \mid q)$ without the superscript + or - , but depending on a scaling parameter $q$, so that

$$
R_{n}(x \mid q)=q^{2 n} R_{n}\left(\frac{x}{q} \mid 1\right)
$$


where $R_{n}\left(\frac{x}{q} \mid 1\right)$ is an $n^{\text {th }}$ order polynomial of $\frac{x}{q}$ with constant coefficients. The $R_{n}(x \mid q)$ are related to $R_{n}^{ \pm}(x)$ defined by (C.23)-(C.25) through

$$
\begin{aligned}
& R_{n}(x \mid q)=R_{n}^{+}(x) \text { when } q=q^{+} \equiv+\frac{i}{2 p} \\
& R_{n}(x \mid q)=R_{n}^{-}(x) \text { when } q=q^{-} \equiv-\frac{i}{2 p} .
\end{aligned}
$$

Writing

$$
u \equiv \frac{x}{q} \quad \text { and } \quad R_{n}\left(\frac{x}{q} \mid 1\right) \equiv r_{n}(u),
$$

we have

$$
\begin{gathered}
r_{0}(u)=1, \quad r_{1}(u)=u, \quad r_{2}(u)=\frac{1}{2} u^{2}+u, \\
r_{3}(u)=\frac{1}{6} u^{3}+u^{2}+2 u, \\
r_{4}(u)=\frac{1}{24} u^{4}+\frac{1}{2} u^{3}+\frac{5}{2} u^{2}+5 u, \quad \text { etc. }
\end{gathered}
$$

correspondingly

$$
\begin{gathered}
R_{0}(x \mid q)=1, \quad R_{1}(x \mid q)=q x, \quad R_{2}(x \mid q)=\frac{1}{2} q^{2} x^{2}+q^{3} x, \\
R_{3}(x \mid q)=\frac{1}{6} q^{3} x^{3}+q^{4} x^{2}+2 q^{5} x, \\
R_{4}(x \mid q)=\frac{1}{24} q^{4} x^{4}+\frac{1}{2} q^{5} x^{3}+\frac{5}{2} q^{6} x^{2}+5 q^{7} x, \text { etc. }
\end{gathered}
$$

In terms of $R_{n}^{ \pm}(x)$, the $P_{n}^{ \pm}(x)$ that satisfies (C.22) is given by

$$
\begin{aligned}
P_{n}^{ \pm}(x)= & \frac{1}{2} \Delta_{1} \Delta_{2} \cdots \Delta_{n} R_{n}^{ \pm}(x) \\
& +c_{1}^{ \pm} \Delta_{2} \Delta_{3} \cdots \Delta_{n} R_{n-1}^{ \pm}(x) \\
& +c_{2}^{ \pm} \Delta_{3} \cdots \Delta_{n} R_{n-2}^{ \pm}(x)+\cdots \\
& +c_{n-1}^{ \pm} \Delta_{n} R_{1}^{ \pm}(x)+c_{n}^{ \pm}
\end{aligned}
$$

where $c_{1}^{ \pm}, c_{2}^{ \pm}, \cdots, c_{n}^{ \pm}$are integration constants, with $c_{m}^{ \pm}=\left(c_{m}^{\mp}\right)^{*}$ for all $m$. Thus for $n=1$, since $R_{1}(x \mid q)=q x$,

$$
R_{1}^{ \pm}(x)=R_{1}\left(x \mid q^{ \pm}\right)=R_{1}\left(x \mid \pm \frac{i}{2 p}\right)= \pm \frac{i x}{2 p} .
$$


According to (C.31),

$$
P_{1}^{ \pm}(x)=\frac{1}{2} \Delta_{1} R_{1}^{ \pm}(x)+c_{1}^{ \pm}= \pm \frac{i x}{4 p} \Delta_{1}+c_{1}^{ \pm}
$$

and from (C.21)

$$
P_{1}=P_{1}^{+}+P_{1}^{-}=c_{1}^{+}+c_{1}^{-}
$$

and

Since

$$
Q_{1}=i\left(P_{1}^{+}-P_{1}^{-}\right)=-\frac{x}{2 p} \Delta_{1}+i\left(c_{1}^{+}-c_{1}^{-}\right) .
$$

$$
\Delta_{1}=2\left(h(x)-\mathcal{E}_{1}\right)=\left\{\begin{array}{lll}
-2 \mathcal{E}_{1} & l<x<L+l, \\
g^{2}-2 \mathcal{E}_{1} & \text { for } & 0<x<l,
\end{array}\right.
$$

we have, in agreement with (C.16), $P_{1}$ is a constant in $l<x<L+l$, and a different constant in $0<x<l$. Likewise,

$$
Q_{1}=\frac{\mathcal{E}_{1} x}{p}+\text { constant } \quad \text { in } l<x<L+l
$$

and

$$
Q_{1}=-\frac{1}{p}\left(\frac{g^{2}}{2}-\mathcal{E}_{1}\right) x+\text { a different constant } \quad \text { in } 0<x<l .
$$

These integration constants are determined by the continuity equations between regions, leaving one final overall constant that is not determined. Likewise, we can derive the solution $f_{n}(x)$ for any $n$.

If we impose the boundary condition at $x=L+l$,

$$
f_{n}(L+l)=1,
$$

then in accordance with the Hierarchy Theorem

$$
\mathcal{E}_{1}<\mathcal{E}_{2}<\cdots
$$

with

$$
\lim _{n \rightarrow \infty} \mathcal{E}_{n}=\mathcal{E}=E_{0}-E
$$

for any finite $g^{2}$. 


\section{C.2 Analyticity of $E\left(g^{2}\right)$}

The convergence of the perturbative series in $g^{2}$ depends on, in the complex $g^{2}$ plane, the location of the nearest singularity $g_{c}^{2}$ of $E\left(g^{2}\right)$ to the origin. While the simple model discussed above can be readily solved, it is more complicated to locate the singularities of $E\left(g^{2}\right)$. We first discuss the exact solution of (C.5). Again we need only consider the positive $x$-axis. Note that the well-depth $-\frac{g^{2}}{2}$ has a critical value $-\frac{g_{0}^{2}}{2}$; for $g^{2}>g_{0}^{2}, E<0$, and for $g^{2}<g_{0}^{2}, E>0$. When $g^{2}=g_{0}^{2}, E=0$ and

$$
\psi=\psi_{c} \propto\left\{\begin{array}{lll}
\frac{L+l-x}{L} & \text { for } & l<x<L+l, \\
\cos q_{0} x & 0<x<l
\end{array}\right.
$$

with

$$
q_{0} \tan q_{0} l=\frac{1}{L}
$$

as required by the continuity of $\psi^{\prime} / \psi$ at $x=l$. When $g^{2}<g_{0}^{2}$,

$$
\psi \propto\left\{\begin{array}{lll}
\sin [k(L+l-x)] & l<x<L+l, \\
\cos q x & \text { for } & 0<x<l
\end{array}\right.
$$

with

$$
q \tan q l=k \cot k L
$$

and

$$
E=\frac{k^{2}}{2}=\frac{1}{2}\left(q^{2}-g^{2}\right)>0 .
$$

When $g^{2}>g_{0}^{2}$,

$$
\psi \propto\left\{\begin{array}{lll}
\sinh [\kappa(L+l-x)] & l<x<L+l, \\
\cos q x & \text { for } & 0<x<l
\end{array}\right.
$$

with

$$
q \tan q l=\kappa \operatorname{coth} \kappa L
$$


and

$$
E=-\frac{\kappa^{2}}{2}=\frac{1}{2}\left(q^{2}-g^{2}\right)<0
$$

In the limit $L \rightarrow \infty, q_{0}=0$, in accordance with (C.41), and therefore

$$
g_{0}^{2}=0 .
$$

For any $g^{2}$ however small, $E$ is negative. Solution (C.45) becomes

$$
\psi \propto\left\{\begin{array}{lll}
e^{-\kappa x} & & x>l, \\
\cos q x & \text { for } & \\
& & x<l
\end{array}\right.
$$

with

$$
E=-\frac{\kappa^{2}}{2}=-\frac{1}{2}\left(g^{2}-q^{2}\right)
$$

and

$$
\kappa=q \tan q l .
$$

We examine the analyticity of $E\left(g^{2}\right)$ for the case $L=\infty$. the same analysis can be extended to arbitrary $L$, but it is more complex. The singularities of $E=E\left(g^{2}\right)$ can be determined by setting $\frac{d E}{d g^{2}}=\infty$; i.e., $\frac{d g^{2}}{d E}=0$, which leads to, on account of $E=-\frac{1}{2} \kappa^{2}$,

$$
\frac{d g^{2}}{d \kappa^{2}}=0
$$

Because $g^{2}=q^{2}+\kappa^{2}$ according to (C.50), (C.52) is equivalent to $\frac{d q^{2}}{d \kappa^{2}}+1=0$; i.e.,

$$
\frac{d q}{d \kappa}=-\frac{\kappa}{q}
$$

Differentiating (C.51), we find

$$
\frac{d \kappa}{d q}=\tan q l+q l \sec ^{2} q l
$$

which, when combined with (C.53), gives

$$
-\frac{q}{\kappa}=\tan q l+q l \sec ^{2} q l .
$$


Since $(q / \kappa)=\cot q l$ according to (C.51), (C.55) becomes

$$
\cot q l+\tan q l+q l \sec ^{2} q l=0 ;
$$

i.e.,

$$
\sec ^{2} q l(\cot q l+q l)=0 .
$$

When $\sec ^{2} q l=0, q l= \pm i \infty$ which is uninteresting for our purpose. Hence, for singularities at a finite $g^{2}$, we require

$$
\cot q l+q l=0,
$$

i.e.,

$$
q l \tan q l=-1 .
$$

Define

$$
z=q l .
$$

Our problem is then reduced to the study of zeroes of

$$
z \tan z+1=0 .
$$

For each zero at $z$, there is a singularity of $E\left(g^{2}\right)$ in the complex $g^{2}$ plane at $g^{2}=\kappa^{2}+q^{2}=q^{2} \tan ^{2} q l+q^{2}$; i.e., at

$$
g^{2} l^{2}=z^{2} \tan ^{2} z+z^{2}=1+z^{2} .
$$

(i) For $z$ imaginary, we set $z= \pm i y$. (C.59) becomes $y \tanh y=1$, which gives for the smallest $|y| \cong 1.2, z \cong \pm 1.2 i$ and

$$
g^{2} l^{2} \cong-0.44 \text {. }
$$

As will be shown below, this gives $g_{c}^{2}$.

(ii) For $z$ real, the nearest zeroes for (C.59) are $z \cong \pm 2.8$ and correspondingly

$$
g^{2} l^{2} \cong 8.8 .
$$

(iii) We shall now establish that in the complex $g^{2}$-plane, the nearest singularity $g_{c}^{2}$ of $E\left(g^{2}\right)$ to the origin is given by (C.61); i.e.,

$$
g_{c}^{2} l^{2} \cong-0.44 \text {. }
$$


Consider the well known expansion

$$
\tan z=2 z \sum_{n=0}^{\infty} \frac{1}{\left(n+\frac{1}{2}\right)^{2} \pi^{2}-z^{2}} .
$$

For $z$ near $\frac{\pi}{2}$, we may write the righthand side as

$$
\begin{aligned}
& 2 z\left[\frac{1}{\left(\frac{\pi}{2}\right)^{2}-z^{2}}+\sum_{n=1}^{\infty} \frac{1}{\left(n+\frac{1}{2}\right)^{2} \pi^{2}-z^{2}}\right] \\
= & 2 z\left[\frac{1}{\left(\frac{\pi}{2}\right)^{2}-z^{2}}+\sum_{n=1}^{\infty}\left(\frac{1}{\left(n+\frac{1}{2}\right)^{2} \pi^{2}}+\frac{z^{2}}{\left(n+\frac{1}{2}\right)^{4} \pi^{4}}+\cdots\right)\right] \\
= & 2 z\left[\frac{1}{\left(\frac{\pi}{2}\right)^{2}-z^{2}}+\left(\frac{1}{2}-\frac{4}{\pi^{2}}\right)+z^{2} \sum_{n=1}^{\infty} \frac{1}{\left(n+\frac{1}{2}\right)^{4} \pi^{4}}+\cdots\right] .
\end{aligned}
$$

By using first the approximation

$$
\tan z \cong 2 z\left[\frac{1}{\left(\frac{\pi}{2}\right)^{2}-z^{2}}+\left(\frac{1}{2}-\frac{4}{\pi^{2}}\right)\right]
$$

and then the correction due to the successive remaining terms in (C.64), one can readily show that (C.61) gives the smallest singularity $g_{c}^{2}$ in the $g^{2}$-plane. (Note that in this case, because $L=\infty, g_{0}=0$ in accordance with (C.48).) Thus, the perturbative series of $E\left(g^{2}\right)$ is convergent only if $g^{2} l^{2}<\left|g_{c}^{2}\right| l^{2} \cong 0.44$. On the other hand, the iterative series of Section C.1 is convergent for any finite $g^{2}$.

\section{C.3 A Comparative Study of Different Green's Functions}

(i) Write the iterative equation (2.19) as

$$
\left(H_{0}-E_{0}\right) \psi_{n}(q)=S_{n}(q)
$$

where

$$
S_{n}(q)=\left(h(q)-\mathcal{E}_{n}\right) \psi_{n-1}(q) .
$$

In one dimension, setting $q=x$ and using (2.25) and (3.15)-(3.16), the solution $\psi_{n}(x)$ is given by

$$
\psi_{n}(x)=\psi_{n}(\infty)+\int_{-\infty}^{\infty}(x|\mathcal{G}| z) S_{n}(z) d z
$$


where the Green's function $\mathcal{G}$ is

$$
(x|\mathcal{G}| z)=-2 \phi(x) \int_{x}^{\infty} \phi^{-2}(y) d y \theta(y-z) \phi(z)
$$

with

$$
\theta(y-z)=\left\{\begin{array}{lll}
1 & & z>y \\
0 & \text { for } & \\
& & z<y
\end{array}\right.
$$

and therefore

$$
(x|\mathcal{G}| z)=0 \quad \text { if } \quad z<x .
$$

The function $(x|\mathcal{G}| z)$ is one of the Sturm-Liouville family of Green's functions[20]. On the other hand, in deriving the usual perturbative series one deals often with similar equations of the form

$$
\left(H_{0}-\lambda\right) u(q)=v(q)
$$

where $\lambda$ is a parameter. Let $u_{n}$ be the $n^{\text {th }}$ member of the ortho-normal set of real eigenfunctions of $H_{0}$ with eigenvalue $e_{n}$. We assume

$$
\int u_{n}(q) u_{m}(q) d^{N} q=\delta_{n m}= \begin{cases}0, & n \neq m, \\ 1, & n=m .\end{cases}
$$

Hence,

$$
\left(H_{0}-e_{n}\right) u_{n}(q)=0
$$

which reduces to (2.8) when $n=0$; i.e.,

$$
e_{0}=E_{0} \quad \text { and } \quad u_{0}=\phi .
$$

For $\lambda$ different from any of the eigenvalues $e_{n}$, the general solution of (C.72) is

$$
u(q)=\int(q|G(\lambda)| \bar{q}) v(\bar{q}) d^{N} \bar{q}
$$

where

$$
(q|G(\lambda)| \bar{q})=\sum_{n} \frac{u_{n}(q) u_{n}(\bar{q})}{e_{n}-\lambda} .
$$


To simplify our notations, we assume all $u_{n}(q)$ to be real. When $\lambda=E_{0}=e_{0}$, (C.72) becomes

$$
\left(H_{0}-e_{0}\right) u(q)=v(q),
$$

same as (C.66). Since $\left(H_{0}-e_{0}\right) u_{0}=0$, we have

$$
\int u_{0}(q) v(q) d^{N} q=0
$$

The general solution of $(\mathrm{C} .78)$ is

$$
u(q)=\int(q|\hat{G}(\lambda)| \bar{q}) v(\bar{q}) d^{N} \bar{q}+c u_{0}(q)
$$

with $c$ an arbitrary constant and

$$
\left(q\left|\hat{G}\left(e_{0}\right)\right| \bar{q}\right) \equiv \sum_{n \neq 0} \frac{u_{n}(q) u_{n}(\bar{q})}{e_{n}-e_{0}} .
$$

In the sub-section (iii) below, we shall analyze the difference between $\mathcal{G}$ and $G$, or $\hat{G}$. Before that, we shall review the roles of $G$ and $\hat{G}$ in the usual perturbative formulas..

(ii) In order to solve the Schroedinger equation (2.4) perturbatively, we begin with (2.13); i.e.,

$$
\left(H_{0}-E_{0}\right) \psi(q)=(h-\mathcal{E}) \psi(q) .
$$

Following the standard Brillouin-Wigner procedure, we require

$$
\int u_{0}(q) \psi(q) d^{N} q=\int \phi(q) \psi(q) d^{N} q=1 .
$$

Define

$$
\hat{\psi}(q)=\psi(q)-u_{0}(q),
$$

which is orthogonal to $u_{0}(q)$, because of (C.83). Eq.(C.82) leads to

$$
\left(H_{0}-E_{0}\right) \hat{\psi}(q)=(h-\mathcal{E})\left(u_{0}(q)+\hat{\psi}(q)\right) ;
$$

on account of (2.12), this gives

$$
\left(H_{0}-E\right) \hat{\psi}(q)=(h-\mathcal{E}) u_{0}(q)+h \hat{\psi}(q) .
$$

Multiply (C.86) on the left by $u_{n}$ and integrate. We have for $n=0$

$$
\mathcal{E}=\int u_{0} h\left(u_{0}+\hat{\psi}\right) d^{N} q
$$


the same as (2.16); for $n \neq 0$

$$
\left(e_{n}-E\right)(n \mid \hat{\psi})=\int u_{n} h\left(u_{0}+\hat{\psi}\right) d^{N} q
$$

where

$$
(n \mid \hat{\psi}) \equiv \int u_{n} \hat{\psi} d^{N} q
$$

Introduce the sequence

$$
\hat{\psi}_{1}, \hat{\psi}_{2}, \hat{\psi}_{3}, \cdots
$$

with

$$
\left(0 \mid \hat{\psi}_{l}\right)=0 \quad \text { for all } \quad l \geq 1 .
$$

When $l=1$, we require for all $n \neq 0$

$$
\left(e_{n}-E\right)\left(n \mid \hat{\psi}_{1}\right)=\int u_{n} h u_{0} d^{N} q .
$$

For $l>1$,

$$
\left(e_{n}-E\right)\left(n \mid \hat{\psi}_{l}\right)=\int u_{n} h \hat{\psi}_{l-1} d^{N} q .
$$

Therefore, the sum

$$
\hat{\psi}=\sum_{1}^{\infty} \hat{\psi}_{l}
$$

satisfies (C.86). In compact form,

$$
\hat{\psi}(q)=\int(q|\hat{G}(E)| \bar{q}) \hat{S}(\bar{q}) d^{N} \bar{q}
$$

where

$$
\hat{S}(q)=h \hat{\psi}(q)+(h-\mathcal{E}) u_{0}(q) .
$$

The usual perturbative series can then be readily derived by first introducing a parameter $\epsilon$, defined by

$$
h(q)=\epsilon \hat{h}(q) .
$$

The subsequent expansions of

$$
\begin{gathered}
E=E_{0}+\epsilon E(1)+\epsilon^{2} E(2)+\cdots \\
\hat{\psi}_{l}=\epsilon \hat{\psi}_{l}(1)+\epsilon^{2} \hat{\psi}_{l}(2)+\cdots
\end{gathered}
$$


and

$$
\begin{aligned}
\hat{G}(E)= & \hat{G}\left(E_{0}\right)+\epsilon E(1) \frac{\partial \hat{G}\left(E_{0}\right)}{\partial E_{0}} \\
& +\epsilon^{2}\left[E(2) \frac{\partial}{\partial E_{0}}+\frac{1}{2}(E(1))^{2} \frac{\partial^{2}}{\partial E_{0}^{2}}\right] \hat{G}\left(E_{0}\right)+\cdots
\end{aligned}
$$

lead to the usual perturbative series.

(iii) According to (C.68)-(C.69), the Green's function $\mathcal{G}$ satisfies

$$
\left(H_{0}-E_{0}\right)(x|\mathcal{G}| z)=\delta(x-z),
$$

whereas the function $\hat{G}\left(E_{0}\right)$ given by (C.81) satisfies, in a one-dimensional problem,

$$
\left(H_{0}-E_{0}\right)\left(x\left|\hat{G}\left(E_{0}\right)\right| z\right)=\delta(x-z)-u_{0}(x) u_{0}(z)
$$

with $u_{0}=\phi$ in accordance with (C.75). Thus $\mathcal{G}$ and $\hat{G}$ are clearly different. As an explicit example, let

$$
H_{0}=-\frac{1}{2} \frac{d^{2}}{d x^{2}}+U(x)
$$

and

$$
U(x)=\left\{\begin{array}{lr}
\infty & x>R \\
0 & \text { for } \quad 0<x<R \\
\infty & x<0
\end{array}\right.
$$

similar to (C.2) and (C.4). The corresponding eigenfunction $u_{n}(x)$ and eigenvalue $e_{n}$ that satisfy (C.73)-(C.74) are

$$
u_{n}=\sqrt{\frac{2}{R}} \sin k_{n} x \quad \text { and } \quad e_{n}=\frac{1}{2} k_{n}^{2}, \quad \text { with } \quad k_{n}=\frac{n+1}{R} \pi,
$$

and $n=0,1,2, \cdots$. For $\lambda$ different from any of the eigenvalues $e_{n}, G(\lambda)$ of (C.77) in this one-dimensional problem is given by

$$
(x|G(\lambda)| z)=\sum_{n=0}^{\infty} \frac{1}{e_{n}-\lambda} u_{n}(x) u_{n}(z)
$$


where $x$ and $z$ can vary between 0 and $R$. Introduce

$$
\begin{gathered}
\chi_{+}=\sin p(R-x), \quad \chi_{-}=\sin p x, \\
\lambda=\frac{1}{2} p^{2}
\end{gathered}
$$

and

$$
\omega=\left(\frac{d \chi_{-}}{d x}\right) \chi_{+}-\left(\frac{d \chi_{+}}{d x}\right) \chi_{-}=p \sin p R .
$$

The Green's function $G(\lambda)$ can also be written as

$$
(x|G(\lambda)| y)=\frac{2}{\omega} \begin{cases}\chi_{-}(x) \chi_{+}(y) & \text { for } \\ \chi_{+}(x) \chi_{-}(y) & x>y .\end{cases}
$$

One can readily verify that $G(\lambda)$ satisfies

$$
\begin{gathered}
(x|G(\lambda)| y)=(y|G(\lambda)| x) \\
\left(-\frac{1}{2} \frac{d^{2}}{d x^{2}}+U(x)\right)(x|G(\lambda)| y)=\delta(x-y)
\end{gathered}
$$

and the boundary conditions

$$
(x|G(\lambda)| 0)=(x|G(\lambda)| R)=0 .
$$

As

$$
\lambda \rightarrow e_{0}=\frac{1}{2}\left(\frac{\pi}{R}\right)^{2},
$$

the Green's function $\hat{G}\left(e_{0}\right)$ of (C.81) is given by

$$
\begin{aligned}
\hat{G}\left(e_{0}\right)= & \lim _{\lambda \rightarrow e_{0}}\left(G(\lambda)-\frac{1}{e_{0}-\lambda} u_{0}(y) u_{0}(x)\right) \\
& =\sum_{n \neq 0} \frac{1}{e_{n}-e_{0}} u_{n}(y) u_{n}(x) .
\end{aligned}
$$

Define

$$
\phi(x)=\sin k_{0} x \quad \text { and } \quad \hat{\phi}(x)=\cos k_{0} x
$$


with $k_{0}=\frac{\pi}{R}$. We find

$$
\begin{array}{rlr}
(x|\hat{G}| z)= & \frac{R}{\pi^{2}} \phi(x) \phi(z)-\frac{2}{\pi}(\phi(x) z \hat{\phi}(z)+\phi(z) x \hat{\phi}(x)) \\
& +\frac{2 R}{\pi} \cdot\left\{\begin{array}{lrr}
\phi(x) \hat{\phi}(z) & x<z, \\
\phi(z) \hat{\phi}(x) & \text { for } & \\
& & x>z .
\end{array}\right.
\end{array}
$$

The function $\mathcal{G}$ can also be expressed in terms of $\phi$ and $\hat{\phi}$. We have

$$
-\frac{R}{\pi} \hat{\phi}(x)=\phi(x) \int_{R / 2}^{x} \frac{d y}{\phi^{2}(y)}
$$

and

$$
(x|\mathcal{G}| z)=\frac{2 R}{\pi} \cdot \begin{cases}\phi(x) \hat{\phi}(z)-\phi(z) \hat{\phi}(x), & x<z, \\ 0, & x>z .\end{cases}
$$

Thus, $\mathcal{G}$ is related, but quite different from $\hat{G}$ and $G$. 